\title{
A Comprehensive Tumor Molecular Profile Analysis in Clinical Practice: A Single Center's Experience
}

\section{Mustafa Özdoğan}

Division of Medical Oncology, Memorial Hospital, Antalya, Turkey

EIRINI PAPADOPOULOU ( $\sim$ eirinipapad@genekor.com )

Genekor Medical S.A, Athens, Greece https://orcid.org/0000-0001-5519-5325

\section{Nikolaos Tsoulos}

Genekor Medical S.A, Athens, Greece

Aikaterini Tsantikidi

Genekor Medical S.A, Athens, Greece

Vasiliki Metaxa-Mariatou

Genekor Medical S.A, Athens, Greece

\section{George Tsaousis}

Genekor Medical S.A, Athens, Greece

\section{Evgenia Kapeni}

Genekor Medical S.A, Athens, Greece

\section{Evgenia Bourkoula}

Genekor Medical S.A, Athens, Greece

\section{Dimitrios Fotiou}

Genekor Medical S.A, Athens, Greece

\section{Georgios Kapetsis}

Genekor Medical S.A, Athens, Greece

loannis Boukovinas

Bioclinic Thessaloniki, Thessaloniki, Greece

Nikolaos Touroutoglou

Department of Medical Oncology, Interbalkan Medical Center, Thessaloniki, Greece

\section{Athanasios Fassas}

St. Luke's Hospital, Thessaloniki, Greece

\section{Achilleas Adamidis}

St. Luke's Hospital, Thessaloniki, Greece

\section{Paraskevas Kosmidis}

Second Department of Medical Oncology, Hygeia Hospital, Athens, Greece

\section{Dimitrios Trafalis}

Henry Dunant Hospital Center

\section{Eleni Galani}


Second Department of Medical Oncology, "Metropolitan" Hospital, Piraeus, Greece.

\section{George Lypas}

Department of Genetic Oncology/Medical Oncology, Hygeia Hospital, Athens, Greece.

\section{Bülent Orhan}

Department of Medical Oncology, Ceylan International Hospital, Bursa, Turkey.

\section{Tansan Sualp}

Tansan Oncology, Istanbul, Turkey.

\section{Tahsin Özatlı}

Istinye University Hospital, Istanbul, Turkey.

\section{Onder Kırca}

Division of Medical Oncology, Memorial Hospital, Antalya, Turkey

\section{Okan Çakır}

Applied Health Sciences, Edinburgh Napier University, EH11 4BN Scotland, United Kingdom

\section{George Nasioulas}

Genekor Medical S.A, Athens, Greece

\section{Research}

Keywords: Molecular profile, Next Generation Sequencing, Targeted treatment, Immunotherapy, Tumor Mutation Burden, PD-L1, Microsatellite instability

Posted Date: October 27th, 2020

DOI: https://doi.org/10.21203/rs.3.rs-96233/v1

License: (9) (i) This work is licensed under a Creative Commons Attribution 4.0 International License. Read Full License 


\section{Abstract}

Background: Tumor molecular profile is of great importance for the detection of biomarkers of response to targeted treatment due to the increased availability, with concomitant reduction of cost, of Next Generation Sequencing technology (NGS). In parallel to targeted therapies', immunotherapies are also evolving, revolutionizing cancer therapy, with Programmed Death-ligand 1 (PD-L1), Microsatellite Instability (MSI), and Tumor Mutational Burden (TMB) analysis being the biomarkers employed most commonly.

Methods: In the present study, a 161 gene NGS panel, containing the majority of clinically significant genes for cancer treatment selection, was used for tumor molecular profile analysis. A variety of tumor types have been analyzed, including aggressive and hard to treat cancers such as pancreatic cancer. Besides, the clinical utility of immunotherapy biomarkers (TMB, MSI, PD-L1), was also studied.

Results: Molecular profile analysis was conducted in 610 cancer patients, while in 393 of them a at least one biomarker for immunotherapy response was requested. At least one actionable alteration was detected in $77.70 \%$ of the patients. $54.59 \%$ of them received information related to on-label or off-label treatment (Tiers 1A.1, 1A.2, 2B, and 2C.1) and $21.31 \%$ received a variant that could be used for clinical trial inclusion. The addition to immunotherapy biomarker to targeted biomarkers' analysis in 191 cases increased the number of patients with an on-label treatment recommendation by $22.40 \%$, while an option for on-label or off-label treatment was provided in $71.35 \%$ of the cases.

Conclusions: Tumor molecular profile analysis by NGS is a first-tier methodology for a variety of tumor types, which provides critical information for treatment decision making in cancer patients. Importantly, simultaneous analysis for targeted therapy and immunotherapy biomarkers could lead to a better tumor characterization and provide actionable information in the majority of patients. Moreover, our results indicate that one in two patients is eligible for ICI treatment based on the biomarkers' analysis. However, when these analyses are performed, the challenge is their implementation in clinical practice. Multidisciplinary patient management is critical to the refinement of the strategy incorporating such information.

\section{Background}

In recent years, technological advances and active research have permitted extensive tumor molecular characterization and have revealed a variety of tumorigenic pathways presenting tumor-specific alterations. These distinctive molecular characteristics of cancer cells can be targeted as they represent the malignant cell's Achille's heel, without affecting the healthy ones. To this regard, of great importance was the previous knowledge gained by large scale studies that used various, advanced technologies to obtain a comprehensive understanding of the tumor molecular profile(1).

Tumor molecular profile is nowadays becoming a reality mainly due to the increased availability, with concomitant reduction of cost of the Next Generation Sequencing technology (NGS) method. The term 
personalized medicine in anticancer treatment has emerged, indicating the need to treat each patient based on his/her tumor's specific characteristics (2). The individualization of treatment strategy entails the use of biomarkers that are those quantifiable characteristics that can be related to cancer prognosis and prediction of treatment response (2-4).

The number of laboratories applying high throughput sequencing analysis is continuously increasing, in parallel with the increased request by the clinicians for such analysis. The frequently insufficient in amount of good quality tissue specimen, coupled with the increasing number of approved targeted agents, make the simultaneous analysis of multiple biomarkers using multigene panels imperative. Thus, advanced technology solves one of the most significant limitations of tissue testing. Of note, optimal paraffin embedding procedure remains crucial for obtaining accurate NGS results $(2,3)$.

Currently, in parallel to targeted therapies, an increasing armamentarium of immunotherapy agents also emerging, revolutionizing cancer therapy. The high cost and toxicity that often accompanies immunotherapeutic agents mandate the use of appropriate biomarkers for selecting patients more likely to benefit from them.

The most widely used biomarker is currently PD-L1 expression, assessed by Immunohistochemistry (IHC) (7). However, it is well known that this is not an ideal biomarker since it is not related to treatment response in many tumor types, while it is clearly not the sole predictor of response to check point inhibition. Moreover, even for those tumors with a proven utility for PDL-1 IHC testing, such as lung cancer, several questions regarding methodology and cut offs remain $(7,8)$.

Additionally, microsatellite instability (MSI) has also been associated with response to anti-PD-L1 treatment with pembrolizumab receiving approval for MSI-H tumors (9-11). Of note, MSI was the first tumor agnostic biomarker that had ever shown efficiency regardless of tumor type. However, the presence of MSI varies among tumor types with the rate of MSI-H tumors ranging from $10-15 \%$ for colon cancer to $0 \%$ in others such as lung cancer (12). Thus, still, the majority of responders will not be identified by it. Hence, the enrichment of biomarkers for the identification of patients eligible for immunotherapy administration is required.

Several additional biomarkers of immune response have been proposed and are currently under investigation while it seems that their combined use could increase the predictive value of the information obtained $(13,14)$. Among the most studied ones is the analysis of Tumor Mutational Burden (TMB) that measures the number of somatic mutations present in a tumor sample. It has been shown in several studies and clinical trials that the greater the number of somatic alterations identified the greater the probability of response to immune treatment (15-17). It has been reported that TMB cutoff values associated with improved survival from immunotherapy treatment vary significantly between cancer types (17). Nevertheless, in the majority of studies and clinical trials, a cut off of 10 muts/MB is used (18-20). Furthermore, the clinical utility of TMB as a predictive biomarker for anti-PD1 treatment administration has been shown in the KEYNOTE 158 study leading to the tumor agnostic approval by the USA FDA of pembrolizumab for metastatic untreatable solid tumors with tissue TMB value of $\geq 10$ muts/MB (21). 
The present study aimed to reveal the applicability and utility of tumor profile analysis in clinical practice, using a pan-cancer NGS panel for cancer treatment selection. The panel used in this study analyses 161 single genes using the Oncomine Technology (Thermo Fischer Scientific) and was selected based on the amount of actionable information contained the robustness of the assay and its relatively low cost which enables its use in clinical practice. A variety of tumor types have been analyzed, including aggressive and hard to treat cancers such as pancreatic cancer. In addition, the clinical utility of immunotherapy biomarkers (TMB, MSI, PD-L1) was also explored.

\section{Methods}

\section{Patients}

In the present study, 629 cancer patients were referred by their treating oncologist for extensive molecular profile analysis from November 2017 to April 2020. Informed consent was obtained from all patients participating in the study. Information concerning sex, age, and tumor histology was obtained, while the pathology report was available in all cases. In addition to molecular analysis for targeted treatment selection, analysis for at least one immunotherapy biomarker (PDL-1, MSI, TMB) was also requested in 395 patients. The analysis was performed using the most recent tissue specimen available.

\section{Tissue selection and nucleic acid isolation}

Genomic DNA and RNA were isolated from formalin-fixed and paraffin-embedded (FFPE) tumor biopsies using the MagMAX ${ }^{\mathrm{TM}}$ Total Nucleic Acid Isolation Kit (Thermo Fischer Scientific) according to the Manufacturer's instructions. The nucleic acid isolation was conducted in the areas of the FFPE block with the majority of tumor cell content (TCC), as indicated by experienced pathologists in Hematoxylin and eosin-stained sections. Minimum required TCC was over $20 \%$, in a tumor area of $>4 \mathrm{~mm}^{2}$.

\section{Next Generation Sequencing}

Whenever tumor molecular profile analysis for targeted therapies was requested, Oncomine Comprehensive Assay v3 (OCAv3) (Thermo Fischer Scientific) was performed, which is an ampliconbased targeted NGS assay. This assay allows the identification of various mutation types such as Single nucleotide Variants (SNVs), insertion-deletions (indels), Copy Number Variations (CNVs), and gene fusions, from 161 unique genes. Run metrics were accessed in the Torrent Suite ${ }^{T M}$ software, using the coverage analysis plugin v5.0.4.0. NGS data analysis was completed with the lon Reporter ${ }^{\mathrm{TM}}$ 5.10.1.0 software (Thermo Fisher Scientific) using the manufacturer's provided workflow (Oncomine Comprehensive v3 - w3.2 - DNA and Fusions - Single Sample). Furthermore, the analysis software Sequence Pilot (version 4.3.0, JSI medical systems, Ettenheim, Germany) was used for variant annotation.

Tumor Mutational Burden analysis was carried out using the Oncomine Tumor Mutation Load Assay (Thermo Fischer Scientific). The assay used is a targeted NGS assay, with 1.65MB of genomic coverage 
(1.2MB exonic) that analyzes 409 genes to provide accurate quantitation of somatic mutations used for tumor mutation burden calculation, in FFPE tissues.

TMB was calculated using the lon reporter pipeline that utilizes a custom variant calling and germline variant filtering to accurately calculate the number of exonic somatic mutations per MB (Oncomine Tumor Mutation Load - w2.0 - DNA - Single Sample).

Microsatellite analysis was conducted using the Ion AmpliSeq ${ }^{\text {TM }}$ Microsatellite Instability Panel (Thermo Fischer Scientific) which is an NGS based assay analyzing 76 markers to assess Microsatellite Instability (MSI) status in tumor-only and tumor-normal samples as indicated by the manufacturer. Analysis of the sequencing output from this panel was carried out using the "MSICall" plugin in the Torrent Suite.

\section{Variant Classification}

Variants were classified according to their predictive value using the four-tiered system jointly recommended by the Association for Molecular Pathology (AMP), the American College of Medical Genetics (ACMG), the American Society of Clinical Oncology (ASCO) and the College of American Pathologists (CAP) for the classification of somatic variants (22). The Joint consensus recommendation system proposed by these major scientific institutions classifies the variants based on their clinical significance in 4 tiers 1-4. Tier 1 variants are of the most substantial clinical significance and are subdivided to those related to sensitivity or resistance to FDA approved treatments (Tier 1A.1), those proposed by professional guidelines to have predictive value (Tier 1A.2), and those with a strong consensus concerning their predictive significance (Tier 1B). The Tier 2 class involves biomarkers with potential clinical relevance. It can be subdivided in variants related to an approved treatment for a different tumor type (Tier 2C.1), variants related to investigational treatments that can be used as an inclusion criterion for patients' enrollment in clinical trials (Tier 2C.1), and variants that have shown predictive value in preclinical studies (Tier 2D). Finally, the 3 and 4 Tiers, include biomarkers of unknown clinical significance and the benign or likely benign ones respectively. $(22,23)$.

\section{Gene panel comparison}

The clinical utility of the 161 gene panel used in this study was evaluated by its comparison to gene panels comprising a smaller gene number. Thus, we simulated the alterations that would have been obtained in our cohort if the analysis was conducted by two hotspot gene panels previously used in our laboratory, a 24 and a 50 gene panel (24) (Additional file 1). Both panels also included the analysis of 6 fusion driver genes ( $A L K, R O S 1, R E T, N T R K 1, N T R K 2$ and NTRK3) analyzed at the RNA level.

Furthermore, in order to investigate if the number of genes analyzed is adequate for implementation in clinical practice, or if by increasing the number of genes tested a more informative result could be obtained, we compared the actionability of the results obtained from this panel to those obtained using a more comprehensive tumor panel that utilizes the same NGS technology. The panel implemented for this evaluation was the Oncomine Comprehensive plus assay (Thermo Fischer Scientific) that analyses the 
full coding sequence of 313 genes, hotspot analysis of 169 genes, CNV of 313 genes (most of them also analyzed for SNV and indels). Furthermore, it includes RNA analysis for 51 fusion drivers genes (38 of them also analyzed at the DNA level), adding up to a total of 514 unique genes present in this panel. The intra-panel comparison was performed through a retrospective analysis of genomic data from The Pancancer Analysis of Whole Genomes (PCAWG) study (25).

The web-based Xena Browser was used for visualization and exploration of the data $(26,27)$. More specifically available data sets from specimens with coding driver alterations information, including single nucleotide variations (SNVs) and small insertions-deletions (indel) and with consensus wholegenome copy number data as well as consensus fusion calls were downloaded and explored. The 990 specimens with information concerning all three types of alterations available were selected.

Subsequently, we simulated the results that would have been obtained if this analysis had been performed using the gene sets included in the aforementioned panels and we explored the magnitude of the clinically actionable information obtained in each case. Variant classification and biomarker interpretation were performed as described above. For the copy number variation analysis, only the 43 genes of the Oncomine Comprehensive Panel v3 and the 333 of the Oncomine comprehensive plus panel with focal copy number variations were included. In order to resemble the cutoff values used in everyday practice in our laboratory, a threshold of $>7$ copies was used for considering a sample positive for copy number amplification and a threshold of $<1$ copy for considering a gene loss (28).

\section{PD-L1 expression by immunohistochemistry}

For the majority of tumors analyzed (such as lung, colorectal, pancreatic and ovarian cancer) as well as for tumors of unknown primary origin, the level of PD-L1 protein expression was defined as the percentage of viable tumor cells (TC) showing partial or complete membrane staining at any intensity. Furthermore, in some cases, the percentage of tumor Infiltrating Immune Cells (IC) showing staining at any intensity was also calculated (29-31). In case of bladder, urothelial, and cervical carcinomas, PD-L1 was calculated through the Combined Positive Score (CPS) which is the percentage of positive cells (tumor, lymphocytes, and macrophages) showing partial or complete membrane staining at any intensity $(32,33)$. In case of Head and Neck Squamous Cell Carcinoma, both CPS and TC values were calculated (34). The analysis was conducted using the Immunohistochemistry (IHC) assay VENTANA PD-L1 (SP263) Assay (Roche Diagnostic) that utilizes the Monoclonal Mouse Anti-PD-L1, Clone SP263 accompanied by OptiView DAB IHC Detection Kit on a VENTANA BenchMark Series automated staining instrument.

For breast cancer patients, the VENTANA Monoclonal Mouse Anti-PD-L1, Clone SP142 antibody was used. The level of expression of the PD-L1 protein was defined as the percentage of tumor-infiltrating Immune Cells showing staining at any intensity (35).

\section{Physicians Survey}

In order to investigate the utility of a multi-biomarker analysis in clinical practice and if the results obtained from such approach have an impact in clinical decision making, a questionnaire was given to 
the referring oncologists, asking whether based on their experience, they consider such analysis useful for patients with the following tumor histological type:

Lung, Colorectal, Breast, Ovarian, Prostate, and rare or unknown origin tumors. It was a multichoice survey with the following options of response: a. Useful, b. in the metastatic setting only c. not useful and d. I do not know/not respond.

\section{Statistical analysis}

Statistics were performed with SPSS (version 20. IBM SPSS STATISTICS). The p-values were based on Fisher's Exact Test. A p-value $<0.05$ was considered to be statistically significant. Box plots were created using the Plotly.js charting library.

\section{Results}

\section{Molecular Analysis for Targeted Therapy}

In the present study, 629 tumor tissues were subjected to targeted treatment biomarkers' analysis, using a 161 gene NGS panel. Successful molecular analysis was achieved in 610 of the 629 patients analyzed, while in 19 (3.03\%) cases, no results could be obtained due to low DNA/RNA quality or quantity. The tumor types analyzed included common tumor types with targeted treatment available, such as lung, breast and colorectal cancer, but also various hard to treat diseases such as pancreatic, ovarian, prostate, brain cancers, sarcomas, cholangiocarcinomas, and others (Figure 1).

The mean age of test requisition was 60 years. In total, 936 pathogenic variants in 112 genes were detected in 472 patients (additional file 2). Of those, $85.15 \%$ were single nucleotide Variants (SNVs) or a small insertions-deletions (indels) detected at the DNA level, while $3.31 \%$ of the variants concerned gene fusions and $11.54 \%$ Copy Number Variations (CNVs). $11.54 \%$ of the 936 variants identified were classified as Tier $1,86.75 \%$ of them as Tier 2 and $1.71 \%$ as Tier 3 (Figure 2). At least one variant was detected in $77.38 \%$ of the cases. $34.98 \%$ of the individuals analyzed carried one genomic alteration, while $23.81 \%$ and $19.87 \%$ carried two and three or more mutations respectively.

The main reason for multigene test request was the assignment of the appropriate treatment based on patients' molecular profile. Thus, patients were apportioned based on the clinical significance of the alterations detected. In the case of multiple mutations present in the same patient, the variant with the higher level of evidence (LOE) was used for establishing the patient's category. Using this biomarkerdefined categorization, $54.59 \%$ of the patients analyzed received information that is related to on-label or off-label treatment (Tiers 1A.1, 1A.2, 1B, and 2C.1). Additionally, the variant detected could be used as a criterion for inclusion in clinical trials (2C.2) or is under investigation in preclinical studies (2D) in $21.48 \%$ and $1.80 \%$ of the cases respectively. Furthermore, $5.90 \%$ of the patients harbored a variant associated with resistance to treatment (1A.1R, 1A.2R) (Figure 3). As expected, the most frequently mutated gene in this cohort was the gatekeeper TP53 gene, followed by the KRAS and PIK3CA genes (Additional file 3). 
These genes were mutated in $36.39 \%, 24.75 \%$ and $10.98 \%$ of the patients, respectively (Figure 4 ). Furthermore, $7.38 \%$ of the patients carried an alteration in a gene involved in the homologous recombination pathway. This type of alterations could be used as predictive biomarkers of response to PARP inhibitors (PARPi) treatment $(36,37)$.

\section{Tissue specific tumor Molecular profile}

In order to evaluate if molecular profile analysis is more useful in specific tumor types compared to others, the mutation frequency and clinical significance of the variants detected were calculated for the most common tumor types analyzed in our cohort.

\section{Pancreatic Cancer}

In the present study, 118 patients undertaking tumor molecular analysis had a diagnosis of pancreatic cancer. KRAS mutation was the prevalent mutated gene in this tumor type, with a mutation frequency of $74.57 \%$. In $64.41 \%$ of the patients, an alteration in this gene was the finding with the higher LoE. However, other gene alterations with predictive value (2C.1) coexisted in $10.16 \%$ of the KRAS mutant patients. Moreover, in 6 cases (5.08\%), the mutation detected was in an HR gene (1 ATM, 2 PALB2, 1 CDK12, 1 $F A N C A, 1 N B M$ ) with evidence of response to PARPi. Additional variants with associated to off-label treatments were detected in FGFR1 \& 4, HER2, MET, PIK3CA and POLE genes (Figure 5, additional file 4).

Furthermore, 2 patients (1.69\%) carried a somatic mutation related to an on-label drug or with strong evidence of actionability. These mutations were detected in genes of the mismatch repair complex (MLH1 and $M S H 2$ ) and were indicative of microsatellite instability and thus response to immunotherapy.

\section{Lung Cancer}

In the 67 Lung cancer, patients tested an alteration was detected in $86.57 \%$ of the cases (figure 5). The variant identified was related to an FDA approved treatment in $20.89 \%$ of the patients. These variants concerned EGFR, BRAF (p.V600) and HER2 mutations in percentages of $8.96 \%, 4.48 \%$ and $1.49 \%$, respectively. Moreover, $A L K$ and $R E T$ translocations were detected in $1.49 \%$ and $4.48 \%$ of the cases, respectively. EGFR TKI resistance-conferring KRAS mutations (Tier 1A.2) were detected in $26.87 \%$ of the cases. Apart from these established biomarkers, the expanded gene panel analysis was able to detect additional mutations in multiple other genes with $2 \mathrm{C} .1$ evidence of predictive value in $16.42 \%$ of the cases (Additional file 5). Unexpectedly, 6 of the patients (8.95\%) carried a mutation in a gene related to PARP inhibitor therapy.

\section{Breast Cancer}

In the 62 Breast Cancer Patients included in our cohort, a pathogenic variant was found in $80.65 \%$ of the cases. A Tier 1 variant was detected in $41.94 \%$ of the patients, while in $9.68 \%$ a Tier $2 \mathrm{C} .1$ variant, related to off-label treatment, was identified. The most prevalent altered gene in these patients was the PIK3CA 
gene, with $33.87 \%$ mutation rate. Additionally, an HR gene alteration was present in $9.68 \%$ of the tumors analyzed (Additional file 6).

\section{Other Cancers}

In the 44 patients with Colorectal cancer, the mutation rate was $84.09 \%$ (Figure 5, additional file 7). Eighteen patients $(40.91 \%)$ carried a mutation in one of the $R A S$ genes which are biomarkers of resistance to EGFR antibodies treatment $(38,39)$. Additionally, three patients carried a targetable BRAF somatic mutation. One PMS2 positive tumor mutation was proven to be of germline origin, and thus it was considered eligible for immunotherapy treatment.

Among the 34 patients with prostate cancer, at least one somatic alteration was identified in $72.73 \%$ of them (Figure 5). In 5 cases, the mutation detected was in an HR gene (14.71\%). Furthermore, $87.88 \%$ of the 33 patients with ovarian cancer, carried at least one somatic alteration. Four patients carried a mutation in BRCA1/2 genes, which are biomarkers of response to PARPi therapy, while in four patients, somatic mutations in off-label biomarkers were identified. Concerning brain tumors, the mutation rate was $77.78 \%$. An alteration with associated potentially significant predictive biomarker was detected in 16 patients (62.96\%) (Figure 5). However, in this tumor histology, the multigene analysis seems to confer not only predictive but also prognostic/diagnostic information $(40,41)$. Genes with diagnostic significance are used by the World Health Organization Classification of Tumors of the Central Nervous System. For example, $I D H 1$ and $I D H 2$ mutations are used for distinguishing primary from secondary gliomas, while the simultaneous presence of $I D H 1 / 2$ and TP53 alterations are distinctive of the diffuse astrocytoma histology (40).

Concerning the other histological types, even if the number of patients tested is small, it seems that in tumors of the endometrium (18 cases), esophagus ( 7 cases) and in cholangiocarcinoma (25 cases) the mutation rate is relatively high $(94.44 \%, 71.43 \%$ and $72.00 \%$ respectively). On the contrary low mutation rates are observed in gastric tumors (30 cases), hepatocellular carcinomas (11 cases) as well as in the 30 sarcomas analyzed $(64.71 \%, 54.55 \%$ and $50.00 \%$ respectively).

\section{Panel comparison}

The genetic information obtained by the 161 gene panel used in this study compared to that obtained from panels containing fewer genes was evaluated. At this regard, we conducted a simulation of the alterations that would have been detected if two smaller hotspot panels, of 24 and 50 genes respectively, had been used in the 610 patients analyzed (additional file 8).

If the 24 gene panel had been used in our cohort, a clinically significant variant (Tier 1 and 2) would have been detected in $58.85 \%$ of the cases. In comparison, this percentage would have been $62.62 \%$ by using the 50 gene panel. However, these rates are much lower than the $77.70 \%$ obtained by the 161 gene panel. Furthermore, considering the on-label and off-label biomarkers, the larger panel managed to detect $14.12 \%$ 
and $10.67 \%$ more on/off-label treatment-related biomarkers compared to the 24 and 50 gene panel respectively (Figure 6).

In order to evaluate if the number of genes analyzed is adequate for implementation in clinical practice, or if by increasing the number of genes tested a more informative result would have been obtained, we compared the actionability of our panel with a more comprehensive panel containing 501 DNA genes and 51 fusion drivers genes (38 of them also analyzed at the DNA level), for a total of 514 unique genes present in this panel (additional file 9).

Among the 990 patients with DNA sequencing results available, an SNV or indel alteration to a driver gene was obtained in $90.4 \%$ (895/990) of the cases using the whole genome sequencing approach of the study. In comparison the 161 gene panel would have detected such alterations in $72.12 \%$ of the patients and the larger panel $83.03 \%$. At least one copy number variations would have been detected in $29.09 \%$ and $47.37 \%$ of the cases by the smaller (161 genes) and bigger panel ( $500+$ genes) respectively. Both panels would have detected a fusion driver gene in $7.68 \%$ of the cases.

Considering all type of alterations (SNV, indel, CNV, gene fusion), at least one actionable alteration would have been identified in $80.00 \%$ of the samples if the 161 gene panel was used and in $90.10 \%$ of them if the 514 gene panel was implemented for the analysis (Figure 7). Furthermore, at least one clinically relevant biomarker, related to on/off-label treatment or to clinical trials would have been detected in $78.28 \%$ and $85.56 \%$ of the cases by the 161 and the 514 gene panels respectively.

Thus, the increase in the number of genes analyzed seems to increase the yield of patients who could benefit from targeted treatments.

\section{Physicians Survey}

Additionally, in order to investigate the implementation of tumor molecular profile analysis among physicians, a questionnaire was sent to referral oncologists asking whether they consider useful, such analysis for treatment decision making in various tumor types. 61 physicians responded to the survey. By far, the tumor type with the majority of positive responses was lung cancer, with $100 \%$ of the physicians responding that multigene panel should be performed for such tumor type (Table 2).

For colorectal cancer patients, a multigene analysis was considered useful in the primary or metastatic setting by $95.08 \%$ of the participants. For breast, ovarian, prostate and pancreatic cancers, the NGS utility was recognized by $80.33 \%, 80.32 \%, 90.16 \%$ and $95.08 \%$ of the participants respectively.

\section{Immunotherapy biomarkers analysis}

Tumor testing can give information for the selection of both appropriate targeted treatment and immunotherapy. The most known immunotherapies biomarkers are TMB, PD-L1 and MSI analysis. In the cohort of 610 patients with successful NGS testing for targeted therapy, 395 also requested TMB analysis. PD-L1 testing was performed in 198 cases, and MSI analysis in 201 patients. In 192 cases, all three 
immunologic biomarkers were analyzed (additional File 10) with successful analysis for all of them achieved in 191 cases.

\section{Tumor Mutation Burden}

Among the 395 patients with TMB analyzed, 14 cases (3.54\%) could not receive a result due to the low quality of the genetic material analyzed. In these cases a high proportion $(>60)$ of variants consistent with de-amination artifacts was detected, and thus these sequencing result could not be evaluated for TMB analysis, as indicated by the manufacturer (42). A successful TMB calculation was obtained for the remaining 381 patients.

The TMB value $\geq 10$ muts/MB has been employed to separate high and low TMB values as indicated by the results of the open-label, phase 2 KEYNOTE-158 study that led to the recent FDA approval of Pembrolizumab for metastatic solid tumors (21). The median TMB value obtained was 5.60 ( $\min 0$; $\max$ 134), with 96 samples showing a TMB value higher than 10 muts/Mb and 285 samples with a lower than 10 muts/Mb value. The tumor type with the highest TMB median value in our cohort was colorectal cancer (median $\mathrm{TMB}=8.02$ ), with 11 samples showing $\mathrm{TMB}>10$ and 21 samples $\mathrm{TMB}<10$ muts/Mb, followed by lung cancer (median $T M B=7.72,25$ samples with $T M B>10$ and 22 with $T M B<10$ muts/Mb (Figure 8). The tumor types with the lowest TMB values were sarcomas, ovarian and pancreatic cancers (median TMB 3.43, 4.44 and 4.63 muts/Mb respectively). Accordingly, the positivity rates varied by tumor type with lung cancer showing the highest (42.55\%) and soft tissue tumors displaying the lowest positivity rate (3.70\%) (Figure 8 ).

\section{PD-L1 expression}

Among the 206 patients referred for PD-L1 analysis by immunohistochemistry, a successful analysis was achieved in 198 cases. PD-L1 positivity (>1\%) was observed in $38.89 \%$ of them (77/988). Moreover, an intense PD-L1 expression was observed in $9.09 \%$ of the patients, exhibiting TPS values greater than $50 \%$ or CPS greater than 50 .

In the 26 lung cancer patients tested $69.23 \%$ had a TPS value $>1$, with $19.23 \%$ showing an intense ( $>50 \%)$ PD-L1 expression. The positivity rate in various tumor types is illustrated in Figure 10. Among the 77 PDL1 positive cases identified in our cohort 26 patients (33.77\%) showed concomitant TMB positivity (>10muts/MB).

In accordance to previous studies, no association of TMB and PD-L1 values was observed (Figure 11) $(43,44)$.

\section{Microsatellite instability}

Microsatellite instability was detected in 8 out of the 206 tumors tested (3.88\%), while for one tumor the analysis failed due to the low quality of the genetic material obtained. Patients with tumors showing MSI high status had a diagnosis of Ovarian cancer, Pancreatic cancer, Colorectal cancer, Prostate cancer, 
Gastric cancer and Sarcoma. In 2 cases, the tumor instability was linked to hereditary mutations in MMR genes (MSH2 and PMS2). TMB analysis data were also available in 7 of these patients with 6 of them showing high TMB value (>13.46muts/MB). Thus a strong correlation between TMB and MSI was observed with MSI high tumors showing higher median TMB values, in accordance with previous studies $(45,46)$. However, it should be noted that among the 193 MSI stable patients with TMB data available, high TMB values were also observed in 42 cases (Figure 11). This is of great importance, given the higher rate of positivity for this biomarker and the strong evidence of predictive value; thus, its use could identify more patients eligible for immunotherapy uptake.

\section{Immunotherapy Biomarkers' comparison}

Among the 191 patients with all three immunotherapy biomarkers tested, ICls option based on TMB result could be considered in 44 patients (23,04\%), 26 of them with simultaneous PD-L1 positivity. Furthermore, 51 additional patients showed PD-L1 positivity and 1 MSI-high result. Collectively, positivity to one of these biomarkers and thus a possibility of benefit from ICls treatment was observed in 50,26\% (96/191) of these patients.

Furthermore, in these patients tested for biomarkers related to both targeted treatment and immunotherapy, an actionable finding (Tier 1 or 2 ) was detected in $83.25 \%$ of the cases. Moreover, the addition of the immunotherapy biomarkers to the molecular profile analysis increased the number of patients with an on-label treatment recommendation by $22.40 \%$ (Figure 12). TMB analysis increased the LoE of treatment recommendations to $1 \mathrm{~A} .1$ in 35 cases, with 22 of them showing concomitant PD-L1 positivity.

\section{Discussion}

\section{Molecular profile analysis}

In the present study, 629 cancer patients have been referred by their treating physician for biomarkers' analysis using a 161 gene NGS panel. In 610 of them, a successful tumor molecular profile was obtained with at least one actionable variant (Tier 1-2)) being detected in $77.70 \%$ of the cases. All pathogenic variants were categorized based on their clinical significance, and only Tier 1 and 2 variants were reported since variants of unknown significance, and the benign/likely benign ones were considered confusing rather than useful for the treatment course information. In $54.59 \%$ of the patients, the information obtained could be used for on-label or off-label treatment reception (Tiers 1A.1, 1A.2, 1B, and 2C.1) while $21.31 \%$ of the cases received a variant that could be used for clinical trials inclusion.

Tests offering comprehensive tumor molecular profiling are currently being requested by a steadily increasing number of oncologists, especially for patients with limited treatment options available. A good implementation of tissue analysis in treatment decision making was observed in the survey conducted in this study among oncologists. More than $80 \%$ of the participating physicians consider clinically useful the tissue NGS analysis for a variety of common tumor types. This percentage was increased to $100 \%$ for 
tumors with many targeted treatment options available such as lung cancer and for tumors with few treatments available such as tumors of unknown origin or rare tumors.

Advances in sequencing technologies and NGS platforms throughput have permitted simultaneous analysis of multiple tumor biomarkers at an adequate time frame to be tailored fit in the design of the treatment plan and at an affordable cost for the patients. The information obtained can be used to address targeted treatment, immunotherapies or in case of negative results traditional treatment approaches. Various studies have shown the efficacy of gene-directed treatment compared to the unselected treatment assignment (47-49). In the IMPACT (Initiative for Molecular Profiling and Advanced Cancer Therapy) study the overall response rate (ORR), and the time-to-treatment failure (TTF) were higher in patients with a molecular aberration that received a matched treatment compared to those who received unmatched treatment (50). Similarly, in the IMPACT/COMPACT trial, the response rate of patients treated according to their genotype had an overall response rate superior compared to those treated on genotype unmatched clinical trials (19\% VS $9 \%$ respectively) (51). Accordingly, the National Comprehensive Cancer Network (NCCN) guidelines outline the contribution of broad molecular profile analysis in the improvement of patients' care in various tumor types. Likewise, the European Society for Medical Oncology (ESMO) recommends routine utilization of tumor NGS analysis for NSCLC, prostate cancer, ovarian cancer and cholangiocarcinoma (52).

Furthermore, more than 200 ongoing clinical trials are currently investigating the impact of molecular directed treatment and the eventual benefit of this approach in patients with several advanced solid tumors and hematological malignancies (www.clinicaltrials.org). Among these, several tumor-agnostic randomized (NCT02152254, NCT03084757) and non-randomized (NCT02465060, NCT03155620, NCT02693535, NCT02290522, NCT03297606, NCT02029001) trials are expected to provide evidence of the clinical benefit of such approach in multiple solid tumor types. Hence, several pharmaceutical companies are focusing on the development of treatments with pan-cancer efficacy (53). The first tumor agnostic therapy with a biomarker included receiving FDA approval was the PD-1 inhibitor Pembrolizumab, which was approved for patients with MSI unstable tumors (53). Subsequently, TRK inhibitor therapy gained approval in NTRK fusion-positive cancers independently from the tumor's histology (54-56). Even though the clinical value of these biomarkers cannot be disputed, the percentage of patients positive for these biomarkers is relatively small. For example, in our study, only 8 out of the 198 patients analyzed presented microsatellite instability. This biomarker seems to be more significant for colorectal cancer patients, where it is present in $10-15 \%$ of the cases, while it is of no use for other tumor types, where it is rarely detected (12). Similarly, the frequency of NTRK fusions in solid tumors of adults is extremely rare in certain tumor types $(54,57)$. Consequently, no positive NTRK tumor was detected in our cohort.

On the other hand, there are agents, with associated biomarkers, that have shown activity in a variety of tumor types. PARP inhibitors are a typical example of such agents having already received approval for Ovarian, Breast, Pancreatic and Prostate cancer patients harboring BRCA1/2 mutations (https://www.fda.gov). Apart from BRCA1/2 mutations, other genes involved in the same pathway of 
homologous recombination seem to be adequate biomarkers of response to such agents, with several clinical trials investigating the expansion of PARPi targeting biomarkers $(36,58-61)$ (www.clinicaltrials.org). These efforts led to the recent approval of the PARP inhibitor Olaparib for metastatic castrate-resistant prostate cancer patients with mutations in other HR genes besides $B R C A 1 / 2$, increasing the percentage of patients with a potential predictive biomarker result who could benefit from that treatment $(59,62)$. Thus, multigene analysis providing comprehensive information about the mutational status of HR genes should be used for better identification of responders to such therapy. In our cohort, $7.38 \%$ of the patients carried an alteration in an HR gene, with certain tumors showing increased levels of these alterations, such as breast cancer (9.68\%), ovarian cancer (20\%) and prostate cancer $(14.71 \%)$. Moreover, the majority $(75.56 \%)$ of the HR-positive patients, carried an HR gene mutation in a non $B R C A 1 / 2$ gene, indicating the necessity of gene panel analysis for the identification of patients eligible for PARPi treatment.

An important issue when a multigene analysis is requested is the number of genes that should be included in such analysis and whether analyzing so many genes is offering more confusion than solutions in the physicians' search for an appropriate targeted treatment option for their patients. Therefore, we simulated the clinically significant variants that could be obtained using panels of various gene number and content. We compared our 161 gene panel with two smaller ones of 24 and 50 genes used in the past years in the patients included in this study. The percentage of positive cases if analyzed with a hotspot 24 and 50 gene panel was $58.85 \%$ and $62.62 \%$ respectively, compared to the $77.07 \%$ obtained using the 161 gene panel. On the contrary, for lung cancer patients, the use of 24 gene panel seems acceptable for analysis since it could identify all biomarkers related to on-label treatments' sensitivity or resistance (46.97\%). Thus, our results indicate that the 24 and 50 gene panels are not adequate for pan-cancer analysis since drug approvals of the recent years recommend the analysis of more biomarkers, with the exception of lung cancer.

A panel analyzing 514 single genes has been recently implemented for tumor analysis in our laboratory. Since we have observed an increase in the rate of patients with a positive tumor finding of at least $10 \%$ in the first 50 samples analyzed, we decided to compare it with the panel used in this study in order to evaluate if it could increase the actionable information obtained by tumor testing analysis. Thus, samples with known genetic profile from PCAWG database were used in order to simulate the percentage of tumor alterations that could be obtained using different size of cancer panels. NGS analysis of single-nucleotide variants (SNVs) and indels, could identify mutations in driver genes in $72 \%$ when the 161 gene panel is used. The utilization of a larger panel slightly increases the number of actionable alterations obtained to $83 \%$.

A result related to on/off-label treatment or to a clinical trial would be obtained in $85.56 \%$ of the cases if the 514 gene panel was used compared to $78.28 \%$ obtained by the 161 gene panel. Thus, both panels seem to give comparable results in terms of the actionable information obtained with the 514 gene panel, including the most actionable biomarkers. The main limitation of this comparison is that the variant calling, and copy number methodologies vary between the targeted assays and the whole genome

Page $15 / 42$ 
methodology used in the PCAWG project. Nevertheless, the increase in the number of clinically significant variants identified when a larger panel is used, reflects what is usually observed in clinical practice.

Despite all the advantages, there is much skepticism concerning the use of a personalized selection of appropriate treatment. A first difficulty in using broad tumor molecular profile analysis for treatment selection is the unavailability in some cases of appropriate tumor tissue to perform the analysis. This could be due to the low quantity/quality of the tissue available or to its inaccessibility in some inoperable tumor types $(63,64)$. It has been shown that among patients enrolled in tumor-directed treatments, only $70-90 \%$ of them had adequate tissue quantity/quality to achieve a successful molecular profile (65). The technology used in our study permits tumor molecular profile analysis from a limited quantity of genetic material. Hence, in our cohort more than $97 \%$ of the tumor samples were successfully analyzed.

Furthermore, such analysis can provide an immense quantity of genetic data that needs to be appropriately analyzed and interpreted. Thus, the role of bioinformatics analysis is becoming major to provide accurate molecular analysis results (66). Moreover, standardization of variant annotation and reporting could facilitate the understanding of the results obtained and increase their reliability. In our experience, in the majority of cases with findings associated to off-label treatments recommendations, the long lasting procedures required for the off-label approval of the suggested treatment from the local National Drug Organization for Medicines ("EOF”), or for clinical trial enrollment, often challenged the utilization of the results, especially in cases with advanced disease, requiring immediate management.

While, it is standard practice to perform accurate pre- and post-test counseling prior to a genetic testing for hereditary cancer susceptibility, this is not the case for somatic mutation analysis (67). However, it is crucial for patients referred for tumor genetic analysis to be accurately informed about the necessity and the possible results obtained by tumor testing. Thus, it would be wise to provide an accurate pre-test counseling, explaining the advantages and limitations of the analysis, with particular regard to avoiding raising too high the expectations, since only a subset of patients will receive useful information. In such a way, a patient's disappointment in case of non-informative results will be reduced, and their post-test management will be facilitated. Furthermore, patients should be informed about the possibility of identifying a variant in a gene with known germline mutations. Especially variant's detected in high percentages $(>40 \%)$, are considered of suspicious germline origin. Since this analysis cannot discriminate between germline and somatic variations, the clarification of a variant's origin requires the analysis of patients' healthy tissue, usually blood or saliva. In our cohort, 17 patients with a family history of cancer, requested blood analysis for suspicious germline variant identified in tissue. In 14 of them (82.35\%) the germline origin of the tissue alteration was confirmed (Additional file 11).

\section{Immunotherapy biomarkers}

Analysis of the tumor's molecular profile useful as it is, it seems to be just another piece of the puzzle, since comprehensive tumor profile should include both biomarkers to guide treatment decision making for both targeted therapy as well as for immunotherapy. Thus, the physician having more biomarkers in his disposition could better comprehend the tumor's biology and decide whether targeted therapy or 
immunotherapy matches better in each case. In our cohort analysis of biomarkers for both immunotherapy and targeted therapy, was requested in 395 patients, with TMB being the most common immunotherapy biomarker requested. All three biomarkers' analysis was successful in 191 cases.

$25.20 \%$ of the 381 patients tested had a TMB value $>10$ muts/MB and thus were eligible for ICl treatment. The median TMB values observed in our population were slightly increased compared to those observed in previous studies $(45,68)$. This could be attributed to methodological differences and to the fact that in the majority of cases the patients analyzed have received more than one treatment lines, commonly chemotherapy, which is known to increase tumor's mutation load (69). Similarly, to our study a TMB positivity rate of $21.1 \%$ was observed in a recent study analyzing immunotherapy biomarkers in 48.782 clinical samples (70). TMB has emerged as a promising biomarker of response to such treatments, and several clinical trials have shown that both blood and tissue samples TMB can effectively be used $(15,17,19,71)$. Moreover, the recent approval of anti-PD1 treatment Pembrolizumab for metastatic cancer patients harboring a TMB value $>10$ mut/MB renders the analysis of such biomarker indispensable for treatment selection strategy.

However, this biomarker has also limitations since TMB calculation methods can differ between different assays, while the gene content of the methodology used seems to affect the TMB values obtained (7274). Furthermore, the cut-off values for this marker are not yet fully established. All these issues are addressed from the International harmonization initiatives led by Friends of Cancer Research (FOCR) and the Qualitätssicherungs-Initiative Pathologie (QuIP) (72-74).

Concerning the other immunotherapy biomarkers, analyzed in this study (PD-L1 and MSI), they could assist in a more accurate patients' selection for treatment with Checkpoint inhibitors. PD-L1 expression, measured by immunohistochemistry methods is the most widely used biomarker and the first to be approved for treatment with checkpoint inhibitors (7). Nevertheless, it is not applicable in many tumor types, and its sensitivity and specificity in identifying patients eligible for immunotherapy have also been questioned $(7,8,19,75-77)$. Moreover, while MSI analysis seems to be an appropriate biomarker, its low incidence in the majority of tumor types limits its clinical utility in the majority of neoplasms. In our cohort microsatellite instability was observed in just $3.88 \%$ of the cases; thus, it cannot stand alone as an immunotherapy biomarker, rendering the addition of other biomarkers indispensable to increase the number of patients who could benefit from such treatments.

The incidence of TMB positivity is superior to that of $\mathrm{MSI}(25.20 \%$ compared to $3.88 \%)$. Furthermore, in $21.88 \%(42 / 193)$ of the MSI stable cases, a TMB value of $>10$ muts/MB was observed; thus, these patients could receive ICl based on the TMB result only. Moreover, no association between TMB and PD-L1 values was observed. This is in agreement with previous studies, indicating lack of association between median values of these biomarkers. However, in accordance to a recent study, a higher TMB positivity rate was observed in the TMB high group. (70). The TMB positivity rate among the PD-L1 positive patients was $33.77 \%(26 / 77)$ compared to $15.79 \%(18 / 114)$ in the PD-L1 negative group $(p=0.005)$. Importantly, it has been reported that patients with positive values for both TMB and PD-L1 could have greater benefit from 
such treatment compared to those showing positivity for only one of these biomarkers $(43,44)$. Collectively, among the 191 patients with all three immunotherapy biomarkers tested, ICls option based on TMB result could be considered in 44 patients (23.04\%), 26 of them with simultaneous PD-L1 positivity.

As it can be seen in the Venn diagram (Figure 13) showing the correlation among these biomarkers in 191 patients tested with all three biomarkers, $50.26 \%$ of the cases had at least one positive biomarker. A positive result for both PD-L1 and TMB was seen in 13.61\% of the cases (with simultaneous MSI high result in 3 cases). In 2 patients concomitant TMB and MSI high values were observed (1.05\%). An additional $35.60 \% \%$ of the patients could receive immunotherapy-based one either TMB or PDL-1 or MSI positivity $(8.38 \%, 26.70 \%, 0.52 \%$ respectively).

The analysis of immunotherapy biomarkers, though, does not seem to be the only determinant of response to $\mathrm{ICl}$, since the tumor mutational status also seems to have a significant influence on the probability of response. For example, several studies have shown the reduced efficacy of ICls in NonSmall Cell Lung cancer patients harboring EGFR mutations and $A L K$ rearrangements $(43,78,79)$. The absence of such targetable alteration could direct the treatment strategy to immunotherapy in these malignancies. In addition, it has been shown that alterations in certain genes, such as KRAS, TP53, MET, $A R I D 1 A$ and others are enriched in immunotherapy responsive patients. Thus, their identification could lead to such treatment option (80-82).

Moreover, alterations in DNA repair genes such as the MMR genes, POLE and HR genes have been shown to have a positive predictive effect and are correlated to increased TMB values (83-86). In contrast, other gene alterations such as JAK1/2 and STK11/LKB1, KEAP1 and PTEN mutations are related to resistance to PD-1 Blockade(80,87-89). Interestingly, in our study, 2 of the patients with TMB high values and one patient with PD-L1 positive result also harbored an STK11 mutation. In none of these cases, immunotherapy response was achieved.

Thus, the addition of immunotherapy biomarkers to tumor molecular profiling seems to be a one-way road in order to achieve a comprehensive tumor characterization and provide the right treatment option for each patient. Moreover, the simultaneous analysis of such biomarkers, leads to the increase of patients with an on-label treatment recommendation by $22.40 \%$. By combining immunotherapy and targeted therapy biomarkers, $71.35 \%$ of the patients analyzed received information related to on-label or off-label treatments. This is obviously improved compared to the $50.52 \%$ of on/off-label biomarkers achieved by analyzing only the molecular profile of the tumor in the same patient cohort.

Nowadays it seems that the tissue is not the issue anymore, since NGS technological advantages permit the simultaneous analysis of many targets from limited tissue material, achieving to analyze up to $97 \%$ of the tissue samples as in the present study. The challenge, though, when these analyses are performed is their implementation in clinical practice. Thus, the results obtained must be appropriately comprehended and adopted for the designation of the treatment selection strategy, which can be achieved through interdiscipline collaboration. To this regard, of great use would be the presence of a multidisciplinary Molecular Tumor Board that could assist in the accurate interpretation of the findings obtained from such 
complex NGS analysis and provide therapeutic recommendations based on all available clinical data for each individual patient (90-92).

\section{Conclusions}

The NGS analysis conducted in this study offered actionable information (Tier1 and 2) in $77.70 \%$ of the 610 patients with tumor molecular profile analysis available. Moreover, simultaneous analysis for targeted therapy and immunotherapy biomarkers resulted in a better tumor characterization and provided actionable information in $83.25 \%$ of the 191 patients tested, with one to two patients being eligible for ICI treatment based on the biomarkers' analysis. Thus, the comprehensive analysis of these biomarkers increased the number of patients with a treatment-related finding and contributed to a more individualized approach for cancer treatment. In conclusion, the present study has shown that the implementation of molecular profiling using appropriate pan-cancer panels in clinical practice is feasible. Of significance, is the appropriate comprehension of the molecular results obtained from such analysis and their proper utilization for designing the treatment selection strategy, which can be achieved through inter-discipline collaboration.

\section{List Of Abbreviations}

Next Generation Sequencing technology (NGS), Immunohistochemistry (IHC), Microsatellite Instability (MSI), Tumor Mutational Burden (TMB), Single nucleotide Variants (SNVs), insertion-deletions (indels), Copy Number Variations (CNVs), Association for Molecular Pathology (AMP), the American College of Medical Genetics (ACMG), the American Society of Clinical Oncology (ASCO) and the College of American Pathologists (CAP), Tumor cells (TC), Immune cells (IC), Immune Checkpoint Inhibitors (ICI), European Society for Medical Oncology (ESMO), National Comprehensive Cancer Network (NCCN), PARP inhibitors (PARPi).

\section{Declarations}

\section{Ethics approval and consent to participate}

The study was approved by the ethical committee of "Bioclinic Thessaloniki" Hospital. All patients gave informed consent for molecular analysis in blood and tissue, in accordance with the Declaration of Helsinki. All patients included in the study have consented for publication of the data generated by the analysis performed.

\section{Consent for publication}

All patients included in the study have consented for publication of the data generated by the analysis performed.

\section{Availability of data and materials}


All data generated or analyzed during this study are included in this published article and its supplementary information files.

\section{Competing interests}

The authors EI, NT, AT, VM, GK, EK, EB, GT, DF and GN declare that are employees in GENEKOR MSA. The other authors declare no conflicts of interest.

\section{Funding}

GENEKOR MSA provided support in the form of salaries for authors El, NT, AT, VM, GK, EK, EB, GT, DF and GN but did not have any additional role in the study design, data collection and analysis, decision to publish, or preparation of the manuscript. The specific roles of these authors are articulated in the 'author contributions' section.

\section{Authors contributions}

MO: Conceptualization, Data curation, Formal analysis, Methodology, Supervision, Writing - original draft, Writing - review \& editing

EP: Conceptualization, Data curation, Formal analysis, Methodology, Supervision, Writing - original draft, Writing - review \& editing

NT: Data curation, Supervision, Writing - review \& editing

AT: Methodology, Writing - review \& editing

VM: Data curation, Writing - review \& editing

GT: Data curation, Writing - review \& editing

EK: Analysis of Data, Writing - review \& editing

EB: Analysis of Data, Writing - review \& editing

DF: Analysis of Data, Writing - review \& editing

GK: Analysis of Data, Writing - review \& editing

IB: Data curation, Writing - review \& editing

NT: Data curation, Review \& editing

AF: Data curation, Review \& editing

AA: Data curation, Review \& editing 
PK: Data curation, Review \& editing

DT: Data curation, Review \& editing

EG: Data curation, Review \& editing

LG: Data curation, Review \& editing

BO: Data curation, Review \& editing

ST: Data curation, Review \& editing

TO: Data curation, Review \& editing

OK: Data curation, Review \& editing

OC: Data curation, Review \& editing

GN: Data curation, Resources, Writing - original draft, Writing - review \& editing

\section{Acknowledgements}

The authors would like to thank all the doctors who participated in this study and all the patients who consented to the use of their genetic material.

\section{References}

1. Nakagawa $H$, Wardell CP, Furuta $M$, Taniguchi $H$, Fujimoto A. Cancer whole-genome sequencing: present and future. Oncogene [Internet]. 2015 Dec 3;34(49):5943-50. Available from: http://www.ncbi.nlm.nih.gov/pubmed/25823020

2. Jürgensmeier JM, Eder JP, Herbst RS. New strategies in personalized medicine for solid tumors: molecular markers and clinical trial designs. Clin Cancer Res [Internet]. 2014 Sep 1;20(17):4425-35. Available from: http://www.ncbi.nlm.nih.gov/pubmed/25183480

3. Garinet S, Laurent-Puig P, Blons H, Oudart J-B. Current and Future Molecular Testing in NSCLC, What Can We Expect from New Sequencing Technologies? J Clin Med [Internet]. 2018 Jun 9;7(6). Available from: http://www.ncbi.nlm.nih.gov/pubmed/29890761

4. Malone ER, Oliva M, Sabatini PJB, Stockley TL, Siu LL. Molecular profiling for precision cancer therapies. Genome Med [Internet]. 2020;12(1):8. Available from:

http://www.ncbi.nlm.nih.gov/pubmed/31937368

5. Srinivasan M, Sedmak D, Jewell S. Effect of fixatives and tissue processing on the content and integrity of nucleic acids. Am J Pathol [Internet]. 2002 Dec;161(6):1961-71. Available from: http://www.ncbi.nlm.nih.gov/pubmed/12466110 
6. Ascierto PA, Bifulco C, Palmieri G, Peters S, Sidiropoulos N. Preanalytic Variables and Tissue Stewardship for Reliable Next-Generation Sequencing (NGS) Clinical Analysis. J Mol Diagn [Internet]. 2019;21(5):756-67. Available from: http://www.ncbi.nlm.nih.gov/pubmed/31251989

7. Kim H, Chung J-H. PD-L1 Testing in Non-small Cell Lung Cancer: Past, Present, and Future. J Pathol Transl Med [Internet]. 2019 Jul;53(4):199-206. Available from: http://www.ncbi.nlm.nih.gov/pubmed/31042863

8. Shen X, Zhao B. Efficacy of PD-1 or PD-L1 inhibitors and PD-L1 expression status in cancer: metaanalysis. BMJ [Internet]. 2018 Sep 10;362:k3529. Available from: http://www.ncbi.nlm.nih.gov/pubmed/30201790

9. Le DT, Uram JN, Wang H, Bartlett BR, Kemberling H, Eyring AD, et al. PD-1 Blockade in Tumors with Mismatch-Repair Deficiency. N Engl J Med [Internet]. 2015 Jun 25;372(26):2509-20. Available from: http://www.ncbi.nlm.nih.gov/pubmed/26028255

10. Le DT, Durham JN, Smith KN, Wang H, Bartlett BR, Aulakh LK, et al. Mismatch repair deficiency predicts response of solid tumors to PD-1 blockade. Science [Internet]. 2017;357(6349):409-13. Available from: http://www.ncbi.nlm.nih.gov/pubmed/28596308

11. Zhao P, Li L, Jiang X, Li Q. Mismatch repair deficiency/microsatellite instability-high as a predictor for anti-PD-1/PD-L1 immunotherapy efficacy. J Hematol Oncol [Internet]. 2019;12(1):54. Available from: http://www.ncbi.nlm.nih.gov/pubmed/31151482

12. Bonneville R, Krook MA, Kautto EA, Miya J, Wing MR, Chen H-Z, et al. Landscape of Microsatellite Instability Across 39 Cancer Types. JCO Precis Oncol [Internet]. 2017;2017. Available from: http://www.ncbi.nlm.nih.gov/pubmed/29850653

13. Walk EE, Yohe SL, Beckman A, Schade A, Zutter MM, Pfeifer J, et al. The Cancer Immunotherapy Biomarker Testing Landscape. Arch Pathol Lab Med [Internet]. 2020 Jun;144(6):706-24. Available from: http://www.ncbi.nlm.nih.gov/pubmed/31714809

14. Signorelli D, Giannatempo P, Grazia G, Aiello MM, Bertolini F, Mirabile A, et al. Patients Selection for Immunotherapy in Solid Tumors: Overcome the Naïve Vision of a Single Biomarker. Biomed Res Int [Internet]. 2019;2019:9056417. Available from: http://www.ncbi.nlm.nih.gov/pubmed/31179334

15. Yarchoan M, Hopkins A, Jaffee EM. Tumor Mutational Burden and Response Rate to PD-1 Inhibition. N Engl J Med [Internet]. 2017;377(25):2500-1. Available from: http://www.ncbi.nlm.nih.gov/pubmed/29262275

16. Campesato LF, Barroso-Sousa R, Jimenez L, Correa BR, Sabbaga J, Hoff PM, et al. Comprehensive cancer-gene panels can be used to estimate mutational load and predict clinical benefit to PD-1 blockade in clinical practice. Oncotarget [Internet]. 2015 Oct 27;6(33):34221-7. Available from: http://www.ncbi.nlm.nih.gov/pubmed/26439694

17. Samstein RM, Lee C-H, Shoushtari AN, Hellmann MD, Shen R, Janjigian YY, et al. Tumor mutational load predicts survival after immunotherapy across multiple cancer types. Nat Genet [Internet]. 2019;51(2):202-6. Available from: http://www.ncbi.nlm.nih.gov/pubmed/30643254 
18. Wu Y, Xu J, Du C, Wu Y, Xia D, Lv W, et al. The Predictive Value of Tumor Mutation Burden on Efficacy of Immune Checkpoint Inhibitors in Cancers: A Systematic Review and Meta-Analysis. Front Oncol [Internet]. 2019;9:1161. Available from: http://www.ncbi.nlm.nih.gov/pubmed/31750249

19. Sholl LM, Hirsch FR, Hwang D, Botling J, Lopez-Rios F, Bubendorf L, et al. The Promises and Challenges of Tumor Mutation Burden as an Immunotherapy Biomarker: A Perspective from the International Association for the Study of Lung Cancer Pathology Committee. J Thorac Oncol [Internet]. 2020 Jun 6; Available from: http://www.ncbi.nlm.nih.gov/pubmed/32522712

20. Galuppini F, Dal Pozzo CA, Deckert J, Loupakis F, Fassan M, Baffa R. Tumor mutation burden: from comprehensive mutational screening to the clinic. Cancer Cell Int [Internet]. 2019;19:209. Available from: http://www.ncbi.nlm.nih.gov/pubmed/31406485

21. Marabelle A, Fakih M, Lopez J, Shah M, Shapira-Frommer R, Nakagawa K, et al. Association of tumour mutational burden with outcomes in patients with advanced solid tumours treated with pembrolizumab: prospective biomarker analysis of the multicohort, open-label, phase 2 KEYNOTE158 study. Lancet Oncol [Internet]. 2020 Sep 10; Available from:

http://www.ncbi.nlm.nih.gov/pubmed/32919526

22. Li MM, Datto M, Duncavage EJ, Kulkarni S, Lindeman NI, Roy S, et al. Standards and Guidelines for the Interpretation and Reporting of Sequence Variants in Cancer: A Joint Consensus Recommendation of the Association for Molecular Pathology, American Society of Clinical Oncology, and College of American Pathologists. J Mol Diagnostics [Internet]. 2017;19(1):4-23. Available from: http://dx.doi.org/10.1016/j.jmoldx.2016.10.002

23. Leichsenring J, Horak P, Kreutzfeldt S, Heining C, Christopoulos P, Volckmar A-L, et al. Variant classification in precision oncology. Int J cancer [Internet]. 2019;145(11):2996-3010. Available from: http://www.ncbi.nlm.nih.gov/pubmed/31008532

24. Tsoulos N, Papadopoulou E, Metaxa-Mariatou V, Tsaousis G, Efstathiadou C, Tounta G, et al. Tumor molecular profiling of NSCLC patients using next generation sequencing. Oncol Rep [Internet]. 2017 Dec;38(6):3419-29. Available from: http://www.ncbi.nlm.nih.gov/pubmed/29130105

25. Campbell PJ, Getz G, Korbel JO, Stuart JM, Jennings JL, Stein LD, et al. Pan-cancer analysis of whole genomes. Nature. 2020;578(7793):82-93.

26. Goldman MJ, Craft B, Hastie M, Repečka K, McDade F, Kamath A, et al. Visualizing and interpreting cancer genomics data via the Xena platform. Nat Biotechnol [Internet]. 2020 Jun;38(6):675-8. Available from: http://www.ncbi.nlm.nih.gov/pubmed/32444850

27. Goldman MJ, Zhang J, Fonseca NA, Cortés-Ciriano I, Xiang Q, Craft B, et al. A user guide for the online exploration and visualization of PCAWG data. Nat Commun [Internet]. 2020 Jul 7;11(1):3400. Available from: http://www.ncbi.nlm.nih.gov/pubmed/32636365

28. Dehghani M, Rosenblatt KP, Li L, Rakhade M, Amato RJ. Validation and Clinical Applications of a Comprehensive Next Generation Sequencing System for Molecular Characterization of Solid Cancer Tissues. Front Mol Biosci [Internet]. 2019;6:82. Available from:

http://www.ncbi.nlm.nih.gov/pubmed/31681791

Page 23/42 
29. Marchetti A, Barberis M, Franco R, De Luca G, Pace MV, Staibano S, et al. Multicenter Comparison of 22C3 PharmDx (Agilent) and SP263 (Ventana) Assays to Test PD-L1 Expression for NSCLC Patients to Be Treated with Immune Checkpoint Inhibitors. J Thorac Oncol. 2017;12(11):1654-63.

30. Villaruz LC, Ancevski Hunter K, Kurland BF, Abberbock S, Herbst C, Dacic S. Comparison of PD-L1 immunohistochemistry assays and response to PD-1/L1 inhibitors in advanced non-small-cell lung cancer in clinical practice. Histopathology [Internet]. 2019 Jan;74(2):269-75. Available from: http://www.ncbi.nlm.nih.gov/pubmed/30105871

31. From the American Association of Neurological Surgeons (AANS), American Society of Neuroradiology (ASNR), Cardiovascular and Interventional Radiology Society of Europe (CIRSE), Canadian Interventional Radiology Association (CIRA), Congress of Neurological and WSO (WSO), Sacks D, Baxter B, Campbell BC V, Carpenter JS, Cognard C, et al. Multisociety Consensus Quality Improvement Revised Consensus Statement for Endovascular Therapy of Acute Ischemic Stroke. Int J Stroke [Internet]. 2018;13(6):612-32. Available from:

http://www.ncbi.nlm.nih.gov/pubmed/29786478

32. Tretiakova M, Fulton R, Kocherginsky M, Long T, Ussakli C, Antic T, et al. Concordance study of PD-L1 expression in primary and metastatic bladder carcinomas: comparison of four commonly used antibodies and RNA expression. Mod Pathol [Internet]. 2018;31(4):623-32. Available from: http://www.ncbi.nlm.nih.gov/pubmed/29271413

33. Ning Y-M, Suzman D, Maher VE, Zhang L, Tang S, Ricks T, et al. FDA Approval Summary: Atezolizumab for the Treatment of Patients with Progressive Advanced Urothelial Carcinoma after Platinum-Containing Chemotherapy. Oncologist [Internet]. 2017;22(6):743-9. Available from: http://www.ncbi.nlm.nih.gov/pubmed/28424325

34. Rasmussen JH, Lelkaitis G, Håkansson K, Vogelius IR, Johannesen HH, Fischer BM, et al. Intratumor heterogeneity of PD-L1 expression in head and neck squamous cell carcinoma. Br J Cancer [Internet]. 2019;120(10):1003-6. Available from: http://www.ncbi.nlm.nih.gov/pubmed/30967647

35. Schmid P, Adams S, Rugo HS, Schneeweiss A, Barrios $\mathrm{CH}$, Iwata $\mathrm{H}$, et al. Atezolizumab and NabPaclitaxel in Advanced Triple-Negative Breast Cancer. N Engl J Med [Internet]. 2018;379(22):2108-21. Available from: http://www.ncbi.nlm.nih.gov/pubmed/30345906

36. Mateo J, Lord CJ, Serra V, Tutt A, Balmaña J, Castroviejo-Bermejo M, et al. A decade of clinical development of PARP inhibitors in perspective. Ann Oncol Off J Eur Soc Med Oncol [Internet]. 2019;30(9):1437-47. Available from: http://www.ncbi.nlm.nih.gov/pubmed/31218365

37. Faraoni I, Graziani G. Role of BRCA Mutations in Cancer Treatment with Poly(ADP-ribose) Polymerase (PARP) Inhibitors. Cancers (Basel) [Internet]. 2018 Dec 4;10(12). Available from: http://www.ncbi.nlm.nih.gov/pubmed/30518089

38. De Roock W, Claes B, Bernasconi D, De Schutter J, Biesmans B, Fountzilas G, et al. Effects of KRAS, BRAF, NRAS, and PIK3CA mutations on the efficacy of cetuximab plus chemotherapy in chemotherapy-refractory metastatic colorectal cancer: a retrospective consortium analysis. Lancet Oncol [Internet]. 2010 Aug;11(8):753-62. Available from: http://www.ncbi.nIm.nih.gov/pubmed/20619739 
39. Douillard J-Y, Oliner KS, Siena S, Tabernero J, Burkes R, Barugel M, et al. Panitumumab-FOLFOX4 treatment and RAS mutations in colorectal cancer. N Engl J Med [Internet]. 2013 Sep 12;369(11):1023-34. Available from: http://www.ncbi.nlm.nih.gov/pubmed/24024839

40. Louis DN, Perry A, Reifenberger G, von Deimling A, Figarella-Branger D, Cavenee WK, et al. The 2016 World Health Organization Classification of Tumors of the Central Nervous System: a summary. Acta Neuropathol [Internet]. 2016;131(6):803-20. Available from: http://www.ncbi.nlm.nih.gov/pubmed/27157931

41. Kristensen BW, Priesterbach-Ackley LP, Petersen JK, Wesseling P. Molecular pathology of tumors of the central nervous system. Ann Oncol Off J Eur Soc Med Oncol [Internet]. 2019;30(8):1265-78. Available from: http://www.ncbi.nlm.nih.gov/pubmed/31124566

42. Chaudhary R, Quagliata L, Martin JP, Alborelli I, Cyanam D, Mittal V, et al. A scalable solution for tumor mutational burden from formalin-fixed, paraffin-embedded samples using the Oncomine Tumor Mutation Load Assay. Transl lung cancer Res [Internet]. 2018 Dec;7(6):616-30. Available from: http://www.ncbi.nlm.nih.gov/pubmed/30505706

43. Rizvi H, Sanchez-Vega F, La K, Chatila W, Jonsson P, Halpenny D, et al. Molecular Determinants of Response to Anti-Programmed Cell Death (PD)-1 and Anti-Programmed Death-Ligand 1 (PD-L1) Blockade in Patients With Non-Small-Cell Lung Cancer Profiled With Targeted Next-Generation Sequencing. J Clin Oncol [Internet]. 2018;36(7):633-41. Available from: http://www.ncbi.nlm.nih.gov/pubmed/29337640

44. Yarchoan M, Albacker LA, Hopkins AC, Montesion M, Murugesan K, Vithayathil TT, et al. PD-L1 expression and tumor mutational burden are independent biomarkers in most cancers. JCI Insight. 2019;4(6).

45. Chalmers ZR, Connelly CF, Fabrizio D, Gay L, Ali SM, Ennis R, et al. Analysis of 100,000 human cancer genomes reveals the landscape of tumor mutational burden. Genome Med [Internet]. 2017;9(1):34. Available from: http://www.ncbi.nlm.nih.gov/pubmed/28420421

46. Luchini C, Bibeau F, Ligtenberg MJL, Singh N, Nottegar A, Bosse T, et al. ESMO recommendations on microsatellite instability testing for immunotherapy in cancer, and its relationship with PD-1/PD-L1 expression and tumour mutational burden: a systematic review-based approach. Ann Oncol Off J Eur Soc Med Oncol [Internet]. 2019;30(8):1232-43. Available from: http://www.ncbi.nlm.nih.gov/pubmed/31056702

47. Wheler JJ, Janku F, Naing A, Li Y, Stephen B, Zinner R, et al. Cancer Therapy Directed by Comprehensive Genomic Profiling: A Single Center Study. Cancer Res [Internet]. 2016;76(13):3690701. Available from: http://www.ncbi.nlm.nih.gov/pubmed/27197177

48. Remon J, Dienstmann R. Precision oncology: separating the wheat from the chaff. ESMO open [Internet]. 2018;3(6):e000446. Available from: http://www.ncbi.nlm.nih.gov/pubmed/30425845

49. Zimmer K, Kocher F, Spizzo G, Salem M, Gastl G, Seeber A. Treatment According to Molecular Profiling in Relapsed/Refractory Cancer Patients: A Review Focusing on Latest Profiling Studies. Comput Struct Biotechnol J. 2019;17:447-53. 
50. Tsimberidou A-M, Iskander NG, Hong DS, Wheler JJ, Falchook GS, Fu S, et al. Personalized medicine in a phase I clinical trials program: the MD Anderson Cancer Center initiative. Clin Cancer Res [Internet]. 2012 Nov 15;18(22):6373-83. Available from:

http://www.ncbi.nlm.nih.gov/pubmed/22966018

51. Stockley TL, Oza AM, Berman HK, Leighl NB, Knox JJ, Shepherd FA, et al. Molecular profiling of advanced solid tumors and patient outcomes with genotype-matched clinical trials: the Princess Margaret IMPACT/COMPACT trial. Genome Med [Internet]. 2016;8(1):109. Available from: http://www.ncbi.nlm.nih.gov/pubmed/27782854

52. Mosele F, Remon J, Mateo J, Westphalen C, Barlesi F, Lolkema M, et al. Journal Pre-proof Recommendations for the use of next-generation sequencing (NGS) for patients with metastatic cancers: A report from the ESMO Precision Medicine Working Group Recommendations for the use of next-generation sequencing (NGS) for patients wi. Ann Oncol [Internet]. 2020;xxx(xxx):1-15. Available from: https://doi.org/10.1016/j.annonc.2020.07.014

53. Pestana RC, Sen S, Hobbs BP, Hong DS. Histology-agnostic drug development - considering issues beyond the tissue. Nat Rev Clin Oncol [Internet]. 2020 Jun 11; Available from: http://www.ncbi.nlm.nih.gov/pubmed/32528101

54. Cocco E, Scaltriti M, Drilon A. NTRK fusion-positive cancers and TRK inhibitor therapy. Nat Rev Clin Oncol [Internet]. 2018;15(12):731-47. Available from: http://www.ncbi.nlm.nih.gov/pubmed/30333516

55. Drilon A, Siena S, Ou S-HI, Patel M, Ahn MJ, Lee J, et al. Safety and Antitumor Activity of the Multitargeted Pan-TRK, ROS1, and ALK Inhibitor Entrectinib: Combined Results from Two Phase I Trials (ALKA-372-001 and STARTRK-1). Cancer Discov [Internet]. 2017;7(4):400-9. Available from: http://www.ncbi.nlm.nih.gov/pubmed/28183697

56. Drilon A, Laetsch TW, Kummar S, DuBois SG, Lassen UN, Demetri GD, et al. Efficacy of Larotrectinib in TRK Fusion-Positive Cancers in Adults and Children. N Engl J Med [Internet]. 2018;378(8):731-9. Available from: http://www.ncbi.nlm.nih.gov/pubmed/29466156

57. Solomon JP, Benayed R, Hechtman JF, Ladanyi M. Identifying patients with NTRK fusion cancer. Ann Oncol Off J Eur Soc Med Oncol [Internet]. 2019;30(Suppl_8):viii16-22. Available from: http://www.ncbi.nlm.nih.gov/pubmed/31738428

58. Yi T, Feng Y, Sundaram R, Tie Y, Zheng H, Qian Y, et al. Antitumor efficacy of PARP inhibitors in homologous recombination deficient carcinomas. Int J cancer [Internet]. 2019;145(5):1209-20. Available from: http://www.ncbi.nlm.nih.gov/pubmed/30666631

59. Mateo J, Porta N, Bianchini D, McGovern U, Elliott T, Jones R, et al. Olaparib in patients with metastatic castration-resistant prostate cancer with DNA repair gene aberrations (TOPARP-B): a multicentre, open-label, randomised, phase 2 trial. Lancet Oncol [Internet]. 2020;21(1):162-74. Available from: http://www.ncbi.nlm.nih.gov/pubmed/31806540

60. Yi M, Dong B, Qin S, Chu Q, Wu K, Luo S. Advances and perspectives of PARP inhibitors. Exp Hematol Oncol [Internet]. 2019;8:29. Available from: http://www.ncbi.nlm.nih.gov/pubmed/31737426 
61. Pilié PG, Gay CM, Byers LA, O'Connor MJ, Yap TA. PARP Inhibitors: Extending Benefit Beyond BRCAMutant Cancers. Clin Cancer Res [Internet]. 2019;25(13):3759-71. Available from: http://www.ncbi.nIm.nih.gov/pubmed/30760478

62. Thomas A, Murai J, Pommier Y. The evolving landscape of predictive biomarkers of response to PARP inhibitors. J Clin Invest [Internet]. 2018;128(5):1727-30. Available from: http://www.ncbi.nIm.nih.gov/pubmed/29664016

63. Do H, Dobrovic A. Sequence artifacts in DNA from formalin-fixed tissues: causes and strategies for minimization. Clin Chem [Internet]. 2015 Jan;61(1):64-71. Available from: http://www.ncbi.nlm.nih.gov/pubmed/25421801

64. Zhang P, Lehmann BD, Shyr Y, Guo Y. The Utilization of Formalin Fixed-Paraffin-Embedded Specimens in High Throughput Genomic Studies. Int J Genomics [Internet]. 2017;2017:1926304. Available from: http://www.ncbi.nlm.nih.gov/pubmed/28246590

65. Nawrocki S. Molecular profiling of tumours for precision oncology - high hopes versus reality. Contemp Oncol (Poznan, Poland) [Internet]. 2018 Mar;22(1A):3-6. Available from: http://www.ncbi.nlm.nih.gov/pubmed/29628787

66. Singer J, Irmisch A, Ruscheweyh H-J, Singer F, Toussaint NC, Levesque MP, et al. Bioinformatics for precision oncology. Brief Bioinform [Internet]. 2019;20(3):778-88. Available from: http://www.ncbi.nlm.nih.gov/pubmed/29272324

67. Gori S, Barberis M, Bella MA, Buttitta F, Capoluongo E, Carrera P, et al. Recommendations for the implementation of BRCA testing in ovarian cancer patients and their relatives. Crit Rev Oncol Hematol [Internet]. 2019 Aug;140:67-72. Available from: http://www.ncbi.nlm.nih.gov/pubmed/31176273

68. Alexandrov LB, Nik-Zainal S, Wedge DC, Aparicio SAJR, Behjati S, Biankin A V, et al. Signatures of mutational processes in human cancer. Nature [Internet]. 2013 Aug 22;500(7463):415-21. Available from: http://www.ncbi.nlm.nih.gov/pubmed/23945592

69. McGranahan N, Furness AJS, Rosenthal R, Ramskov S, Lyngaa R, Saini SK, et al. Clonal neoantigens elicit T cell immunoreactivity and sensitivity to immune checkpoint blockade. Science [Internet]. 2016 Mar 25;351(6280):1463-9. Available from: http://www.ncbi.nlm.nih.gov/pubmed/26940869

70. Huang RSP, Haberberger J, Severson E, Duncan DL, Hemmerich A, Edgerly C, et al. A pan-cancer analysis of PD-L1 immunohistochemistry and gene ampli fi cation, tumor mutation burden and microsatellite instability in 48,782 cases. Mod Pathol [Internet]. Available from: http://dx.doi.org/10.1038/s41379-020-00664-y

71. Gandara DR, Paul SM, Kowanetz M, Schleifman E, Zou W, Li Y, et al. Blood-based tumor mutational burden as a predictor of clinical benefit in non-small-cell lung cancer patients treated with atezolizumab. Nat Med [Internet]. 2018;24(9):1441-8. Available from: http://www.ncbi.nlm.nih.gov/pubmed/30082870

72. Stenzinger A, Allen JD, Maas J, Stewart MD, Merino DM, Wempe MM, et al. Tumor mutational burden standardization initiatives: Recommendations for consistent tumor mutational burden assessment in 
clinical samples to guide immunotherapy treatment decisions. Genes Chromosomes Cancer [Internet]. 2019;58(8):578-88. Available from: http://www.ncbi.nlm.nih.gov/pubmed/30664300

73. Merino DM, McShane LM, Fabrizio D, Funari V, Chen S-J, White JR, et al. Establishing guidelines to harmonize tumor mutational burden (TMB): in silico assessment of variation in TMB quantification across diagnostic platforms: phase I of the Friends of Cancer Research TMB Harmonization Project. J Immunother cancer [Internet]. 2020 Mar;8(1). Available from: http://www.ncbi.nIm.nih.gov/pubmed/32217756

74. Stenzinger A, Endris V, Budczies J, Merkelbach-Bruse S, Kazdal D, Dietmaier W, et al. Harmonization and Standardization of Panel-Based Tumor Mutational Burden Measurement: Real-World Results and Recommendations of the Quality in Pathology Study. J Thorac Oncol [Internet]. 2020 Jul;15(7):117789. Available from: http://www.ncbi.nlm.nih.gov/pubmed/32119917

75. Sunshine J, Taube JM. PD-1/PD-L1 inhibitors. Curr Opin Pharmacol [Internet]. 2015 Aug;23:32-8. Available from: http://www.ncbi.nlm.nih.gov/pubmed/26047524

76. Cottrell TR, Taube JM. PD-L1 and Emerging Biomarkers in Immune Checkpoint Blockade Therapy. Cancer J [Internet]. 24(1):41-6. Available from: http://www.ncbi.nlm.nih.gov/pubmed/29360727

77. Apolo AB. PDL1: The Illusion of an Ideal Biomarker. Eur Urol Focus [Internet]. 2016 Feb;1(3):269-71. Available from: http://www.ncbi.nlm.nih.gov/pubmed/28723398

78. Lee CK, Man J, Lord S, Cooper W, Links M, Gebski V, et al. Clinical and Molecular Characteristics Associated With Survival Among Patients Treated With Checkpoint Inhibitors for Advanced Non-Small Cell Lung Carcinoma: A Systematic Review and Meta-analysis. JAMA Oncol [Internet]. 2018 Feb 1;4(2):210-6. Available from: http://www.ncbi.nlm.nih.gov/pubmed/29270615

79. Gainor JF, Shaw AT, Sequist L V, Fu X, Azzoli CG, Piotrowska Z, et al. EGFR Mutations and ALK Rearrangements Are Associated with Low Response Rates to PD-1 Pathway Blockade in Non-Small Cell Lung Cancer: A Retrospective Analysis. Clin Cancer Res [Internet]. 2016 Sep 15;22(18):4585-93. Available from: http://www.ncbi.nlm.nih.gov/pubmed/27225694

80. Skoulidis F, Goldberg ME, Greenawalt DM, Hellmann MD, Awad MM, Gainor JF, et al. STK11/LKB1 Mutations and PD-1 Inhibitor Resistance in KRAS-Mutant Lung Adenocarcinoma. Cancer Discov [Internet]. 2018;8(7):822-35. Available from: http://www.ncbi.nlm.nih.gov/pubmed/29773717

81. Kauffmann-Guerrero D, Tufman A, Kahnert K, Bollmann BA, Reu S, Syunyaeva Z, et al. Response to Checkpoint Inhibition in Non-Small Cell Lung Cancer with Molecular Driver Alterations. Oncol Res Treat [Internet]. 2020;43(6):289-98. Available from: http://www.ncbi.nlm.nih.gov/pubmed/32268332

82. Okamura R, Kato S, Lee S, Jimenez RE, Sicklick JK, Kurzrock R. ARID1A alterations function as a biomarker for longer progression-free survival after anti-PD-1/PD-L1 immunotherapy. J Immunother cancer [Internet]. 2020 Feb;8(1). Available from: http://www.ncbi.nlm.nih.gov/pubmed/32111729

83. Kim JH, Kim SY, Baek JY, Cha YJ, Ahn JB, Kim HS, et al. A Phase II Study of Avelumab Monotherapy in Patients with Mismatch Repair-Deficient/Microsatellite Instability-High or POLE-Mutated Metastatic or Unresectable Colorectal Cancer. Cancer Res Treat [Internet]. 2020 Apr 24; Available from: http://www.ncbi.nlm.nih.gov/pubmed/32340084 
84. Picard E, Verschoor CP, Ma GW, Pawelec G. Relationships Between Immune Landscapes, Genetic Subtypes and Responses to Immunotherapy in Colorectal Cancer. Front Immunol [Internet]. 2020;11:369. Available from: http://www.ncbi.nlm.nih.gov/pubmed/32210966

85. Peyraud F, Italiano A. Combined PARP Inhibition and Immune Checkpoint Therapy in Solid Tumors. Cancers (Basel) [Internet]. 2020 Jun 9;12(6). Available from: http://www.ncbi.nlm.nih.gov/pubmed/32526888

86. Pellegrino B, Musolino A, Llop-Guevara A, Serra V, De Silva P, Hlavata Z, et al. Homologous Recombination Repair Deficiency and the Immune Response in Breast Cancer: A Literature Review. Transl Oncol [Internet]. 2020 Feb;13(2):410-22. Available from: http://www.ncbi.nlm.nih.gov/pubmed/31901781

87. Shin DS, Zaretsky JM, Escuin-Ordinas H, Garcia-Diaz A, Hu-Lieskovan S, Kalbasi A, et al. Primary Resistance to PD-1 Blockade Mediated by JAK1/2 Mutations. Cancer Discov [Internet]. 2017;7(2):188-201. Available from: http://www.ncbi.nlm.nih.gov/pubmed/27903500

88. Zaretsky JM, Garcia-Diaz A, Shin DS, Escuin-Ordinas H, Hugo W, Hu-Lieskovan S, et al. Mutations Associated with Acquired Resistance to PD-1 Blockade in Melanoma. N Engl J Med [Internet]. 2016 Sep 1;375(9):819-29. Available from: http://www.ncbi.nlm.nih.gov/pubmed/27433843

89. Piro G, Carbone C, Carbognin L, Pilotto S, Ciccarese C, lacovelli R, et al. Revising PTEN in the Era of Immunotherapy: New Perspectives for an Old Story. Cancers (Basel) [Internet]. 2019 Oct 10;11(10). Available from: http://www.ncbi.nlm.nih.gov/pubmed/31658667

90. Knepper TC, Bell GC, Hicks JK, Padron E, Teer JK, Vo TT, et al. Key Lessons Learned from Moffitt's Molecular Tumor Board: The Clinical Genomics Action Committee Experience. Oncologist [Internet]. 2017;22(2):144-51. Available from: http://www.ncbi.nlm.nih.gov/pubmed/28179575

91. van der Velden DL, van Herpen CML, van Laarhoven HWM, Smit EF, Groen HJM, Willems SM, et al. Molecular Tumor Boards: current practice and future needs. Ann Oncol Off J Eur Soc Med Oncol [Internet]. 2017 Dec 1;28(12):3070-5. Available from:

http://www.ncbi.nlm.nih.gov/pubmed/29045504

92. Kato S, Kim KH, Lim HJ, Boichard A, Nikanjam M, Weihe E, et al. Real-world data from a molecular tumor board demonstrates improved outcomes with a precision N-of-One strategy. Nat Commun [Internet]. 2020;11(1):4965. Available from: http://www.ncbi.nlm.nih.gov/pubmed/33009371

\section{Tables}


Table 1

Oncologists responses concerning the clinical utility of NGS multigene analysis in various tumor types.

\begin{tabular}{|llllllll|}
\hline $\begin{array}{l}\text { Oncologists } \\
\text { Responses }\end{array}$ & \multicolumn{2}{l}{ Tumor Histology } & & & & & \\
\cline { 2 - 8 } & Lung & Colorectal & Breast & Ovarian & Pancreas & Prostate & $\begin{array}{l}\text { Rare } \\
\text { /Unknown }\end{array}$ \\
\hline Useful & $60.66 \%$ & $65.57 \%$ & $45.90 \%$ & $65.57 \%$ & $50.82 \%$ & $50.82 \%$ & $85.25 \%$ \\
\hline $\begin{array}{l}\text { Useful in the } \\
\text { metastatic } \\
\text { setting }\end{array}$ & $39.34 \%$ & $29.51 \%$ & $34.43 \%$ & $14.75 \%$ & $44.26 \%$ & $39.34 \%$ & $14.75 \%$ \\
\hline $\begin{array}{l}\text { Not useful } \\
\begin{array}{l}\text { No } \\
\text { opinion/response }\end{array}\end{array}$ & $0.00 \%$ & $4.92 \%$ & $9.84 \%$ & $9.84 \%$ & $0.00 \%$ & $0.00 \%$ & $0.00 \%$ \\
\hline
\end{tabular}

\section{Figures}

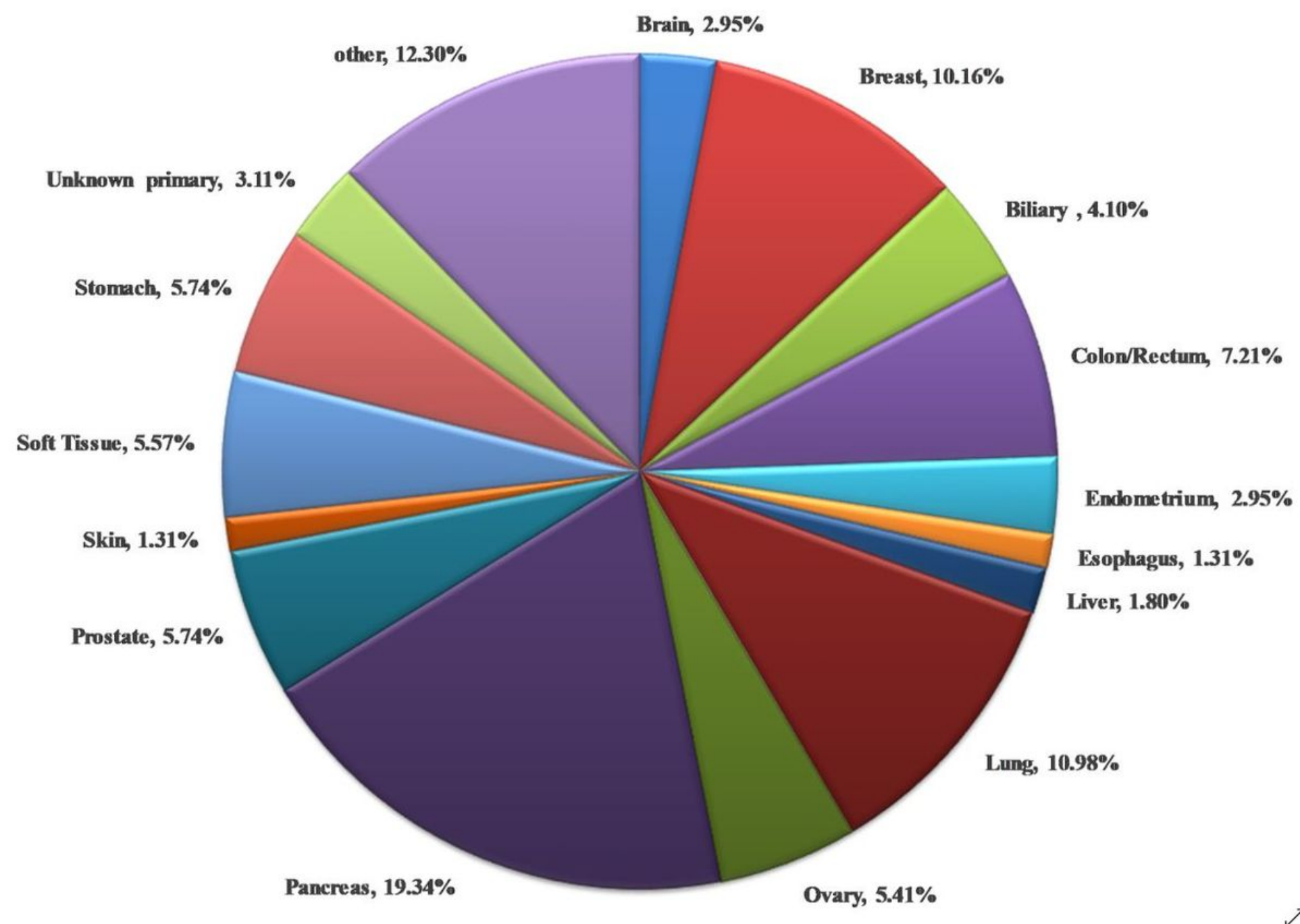

Figure 1 
Tumor histological types analyzed by NGS.

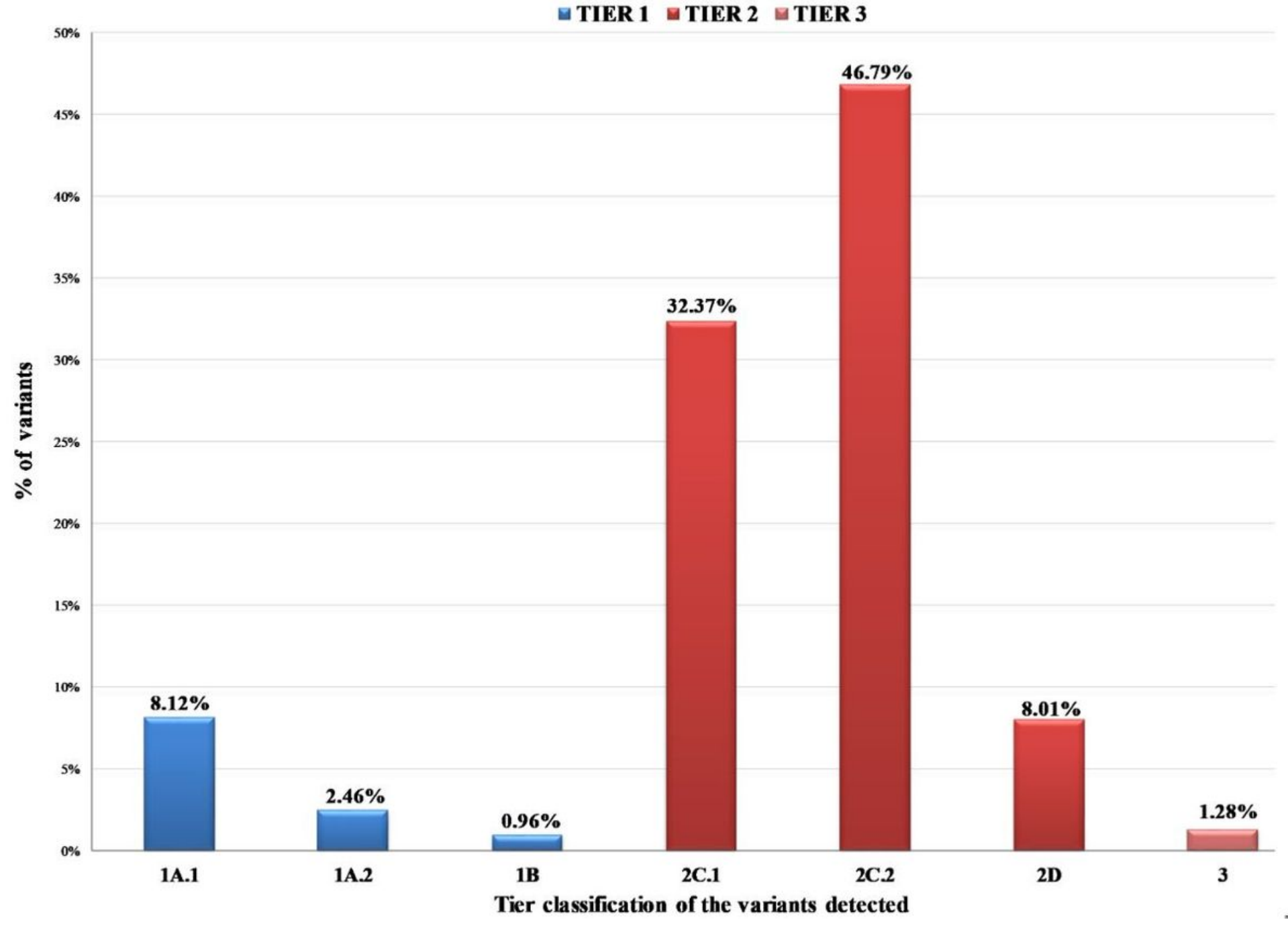

\section{Figure 2}

Tier Classification of the 936 pathogenic variants identified in the 610 tumors analyzed based on their predictive value. 1A.1: Biomarkers related to on-label treatment, 1A.2: Biomarkers included in guidelines, 1B: Biomarkers with strong evidence of correlation to treatment, 2C.1: Biomarkers related to off-label treatment, 2C.1: Biomarkers related to clinical trials, 2D: Biomarkers with preclinical evidence of actionability, 3: Biomarkers with unknown actionability. 


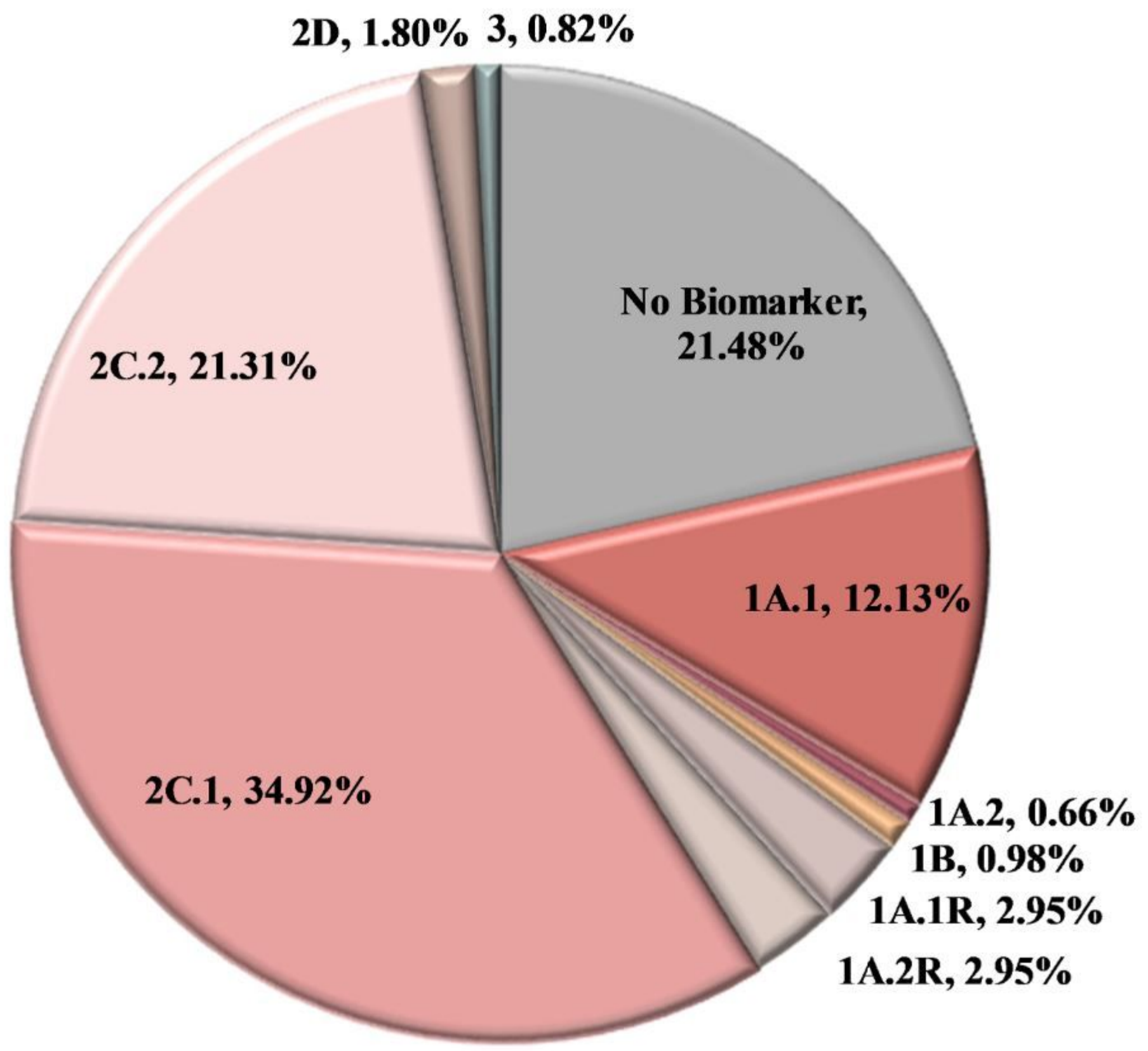

Figure 3

Patients categorization in the entire cohort based on the most clinically significant variant detected. In the case of multiple mutations present in the same patient, the variant with the higher level of evidence was used for establishing patient's category. 1A.1: Biomarkers related to on-label treatment, 1A.1R: Biomarkers related to resistance to an on-label treatment, 1A.2: Biomarkers included in guidelines, 1A.2R: Biomarkers related to resistance in a treatment included in guidelines, $1 \mathrm{~B}$ : Biomarkers with strong evidence of correlation to treatment, 2C.1: Biomarkers related to off-label treatment, 2C.1: Biomarkers related to clinical trials, 2D: Biomarkers with preclinical evidence of actionability, 3:Biomarkers with unknown actionability, No Biomarker: Patients with no biomarker available. 


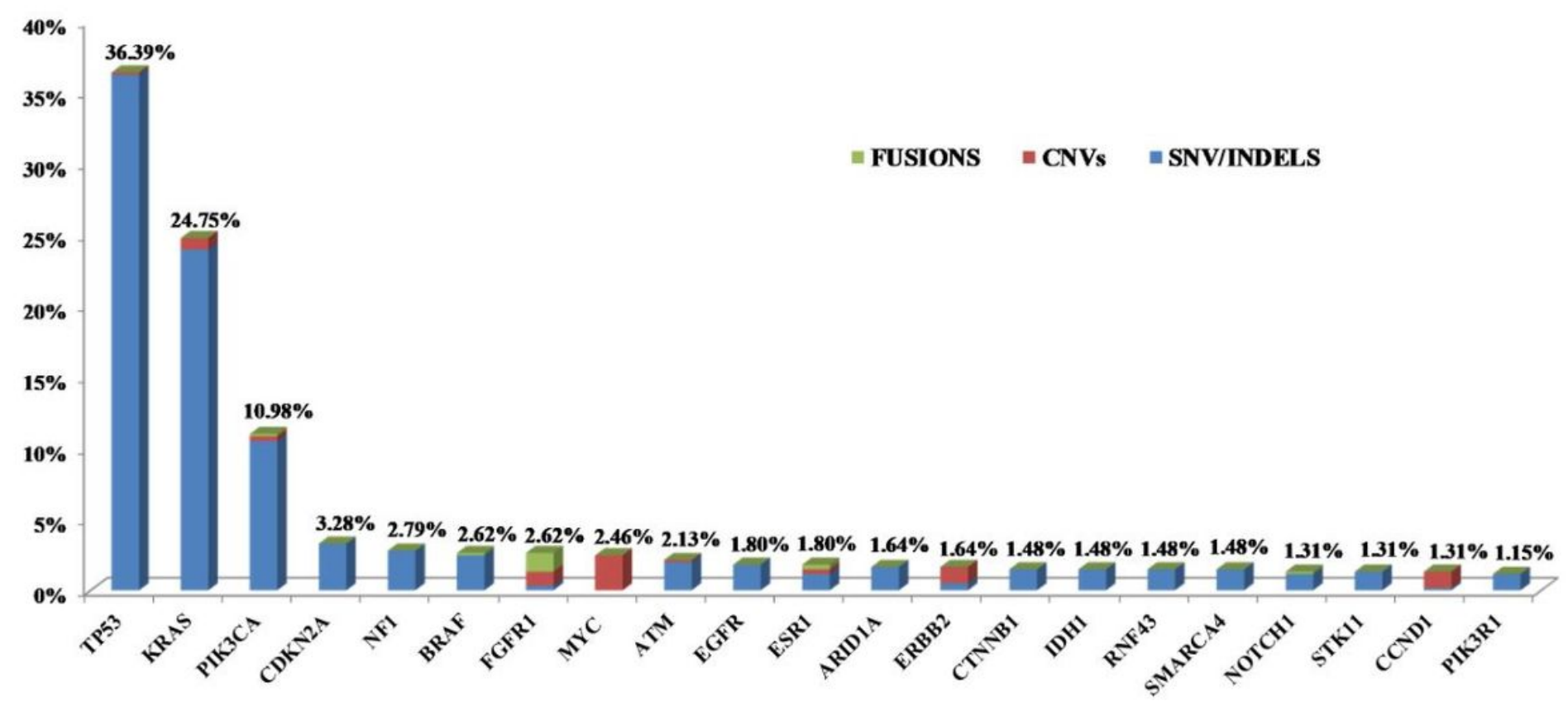

Figure 4

The Top 20 most frequently altered genes in the cohort analyzed.

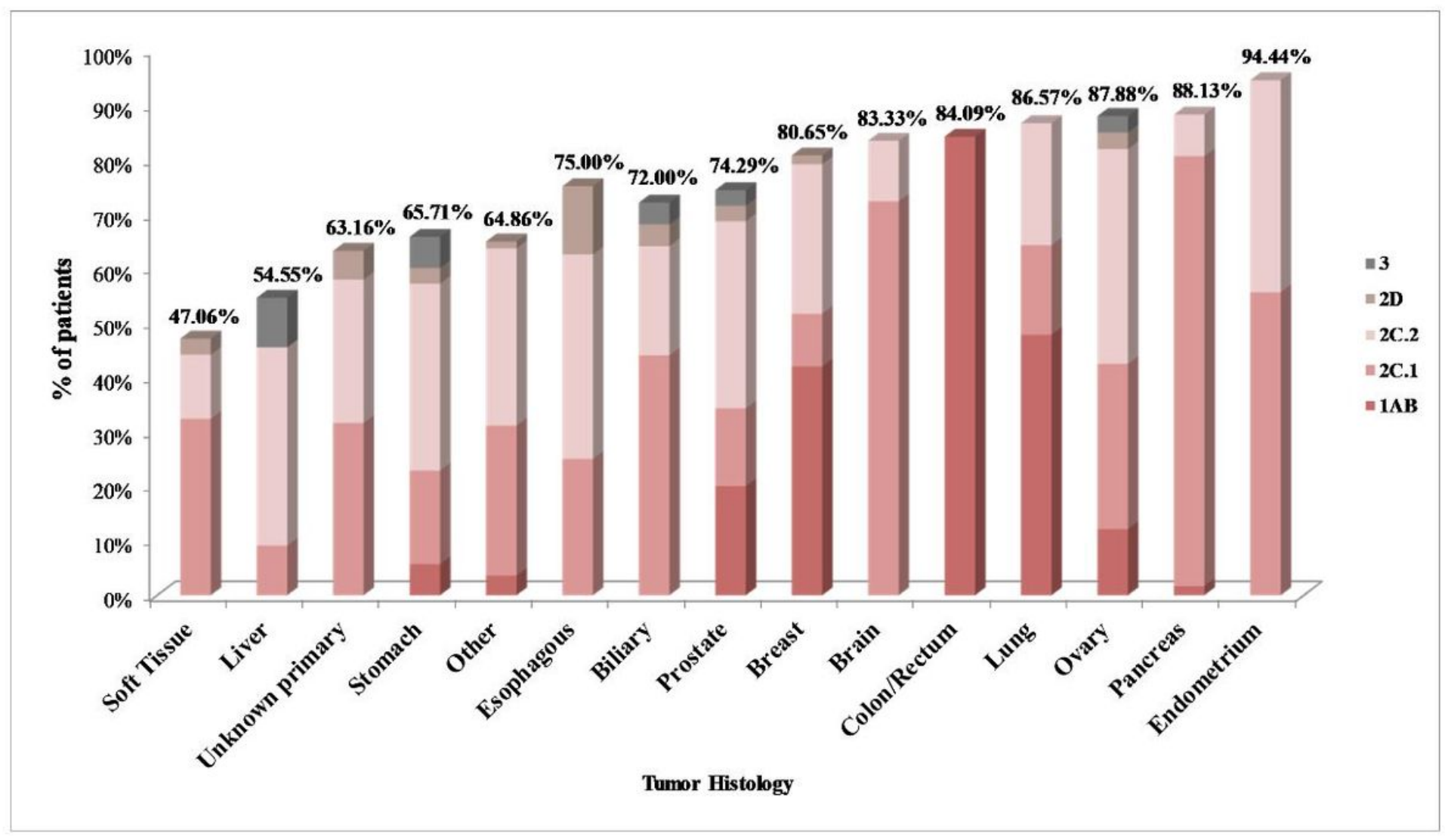

Figure 5 
Mutation Rate and patients' categorization based on the Tier classification of the most clinically significant variant for each tumor histology. The following categories were used: 1A/1B: Patients harboring alterations that are biomarkers for on-label treatments or with strong evidence of predictive value for an on-label treatment (Tier 1), 2C.1: Patients with biomarkers related to off-label treatment, 2C.2: Patients with biomarkers related to clinical trials, 2D: Patients with biomarkers with preclinical evidence of predictive value, 3: Patients harboring alterations with conflicting evidence of cancer association.

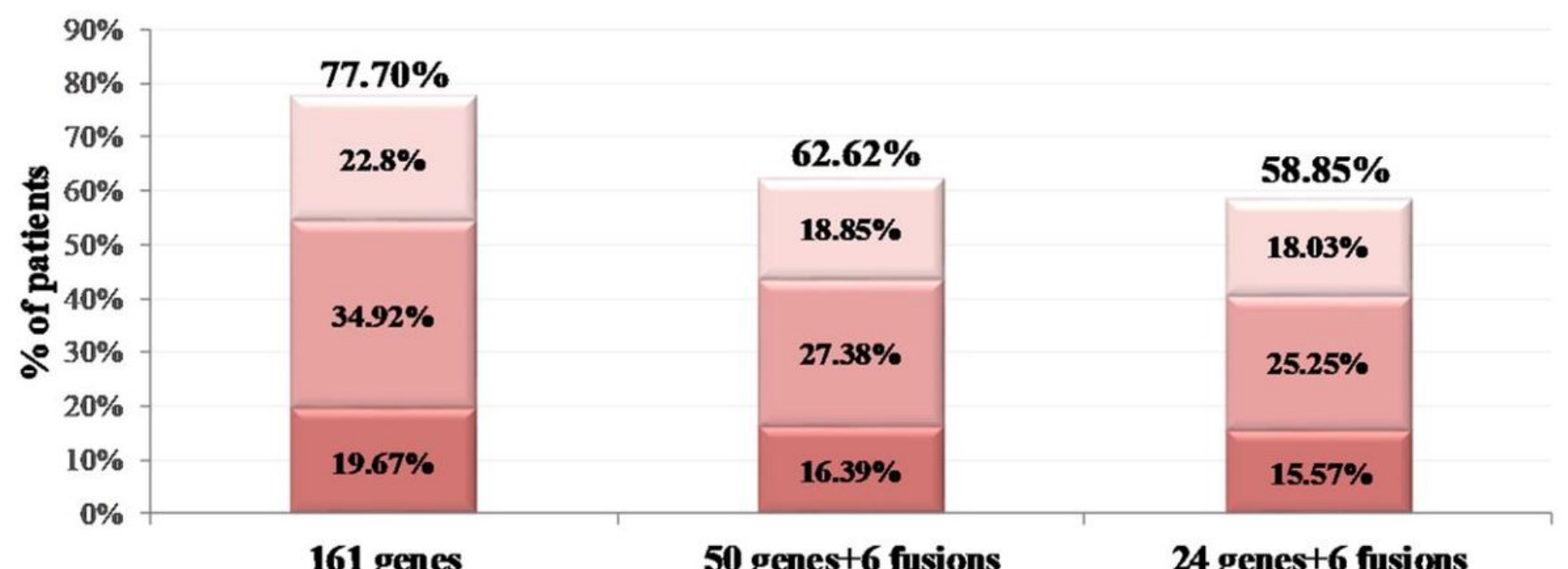

$\square$ Tier $1 \square 2 C .1 \square 2 C .2 / 2 D$

Figure 6

Simulation of patients' biomarker-defined categorization based on their most clinically significant variant when the analysis is performed using either the 161 or the 50 or the 24 gene panels. The following categories were used: 1A/1B: Patients carrying Tier 1 alterations, 2C.1: Patients with 2C.1 alterations, 2C.2/2D Patients with $2 \mathrm{C} .2$ or $2 \mathrm{D}$ alterations. 


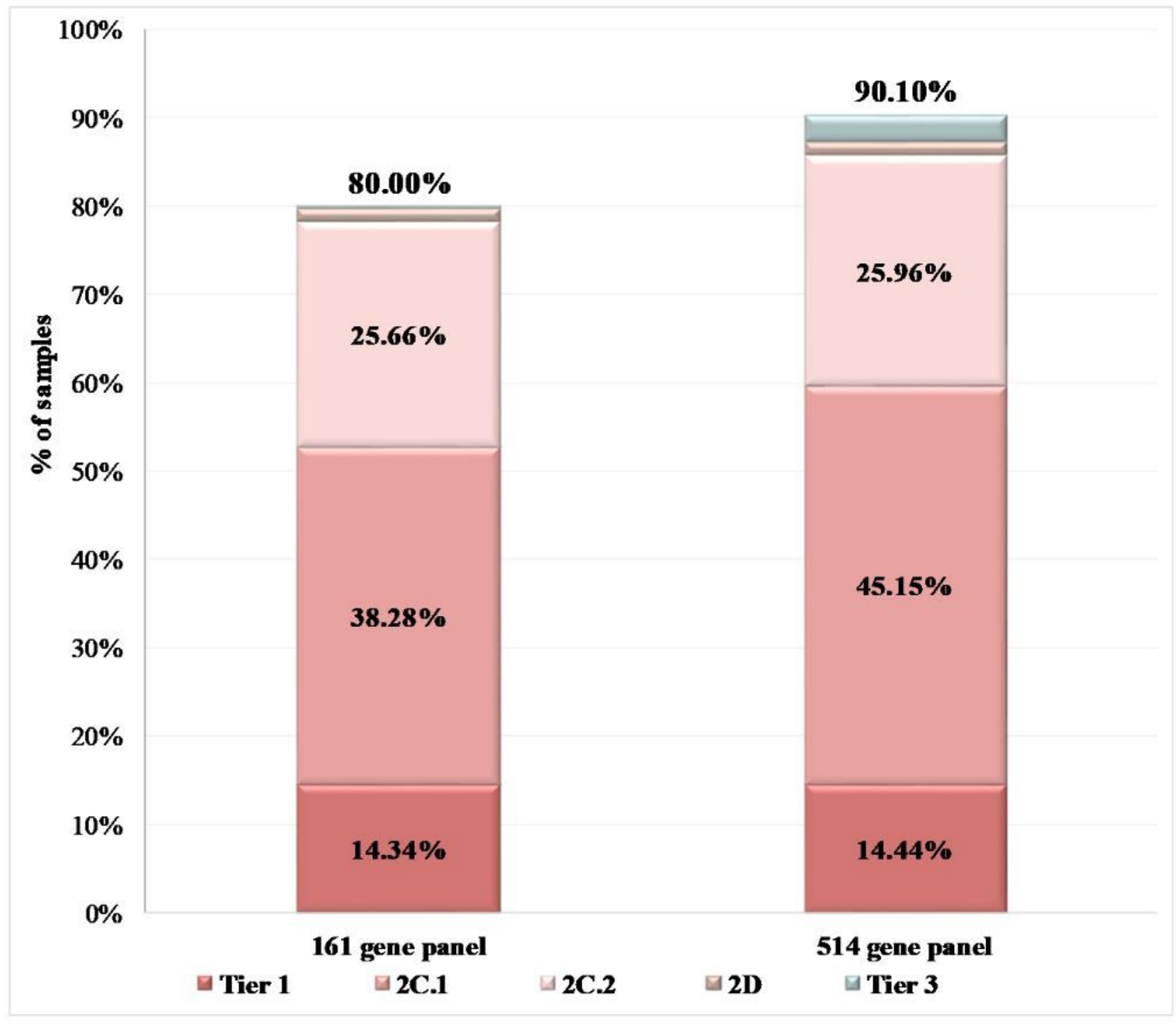

Figure 7

Simulation of the 990 PCAWG samples' categorization based on their most clinically significant variant when the analysis is performed using either the 161 the 514 gene panels. The following categories were used: Tier 1 variants: Patients carrying at least one Tier 1 alteration, 2C.1: Patients whose most clinically significant variant is classified as 2C.1, 2C.2: Patients with 2C.2 alterations, 2D: Patients with 2D alterations, Tier 3: Patients carrying Tier 3 alterations. 


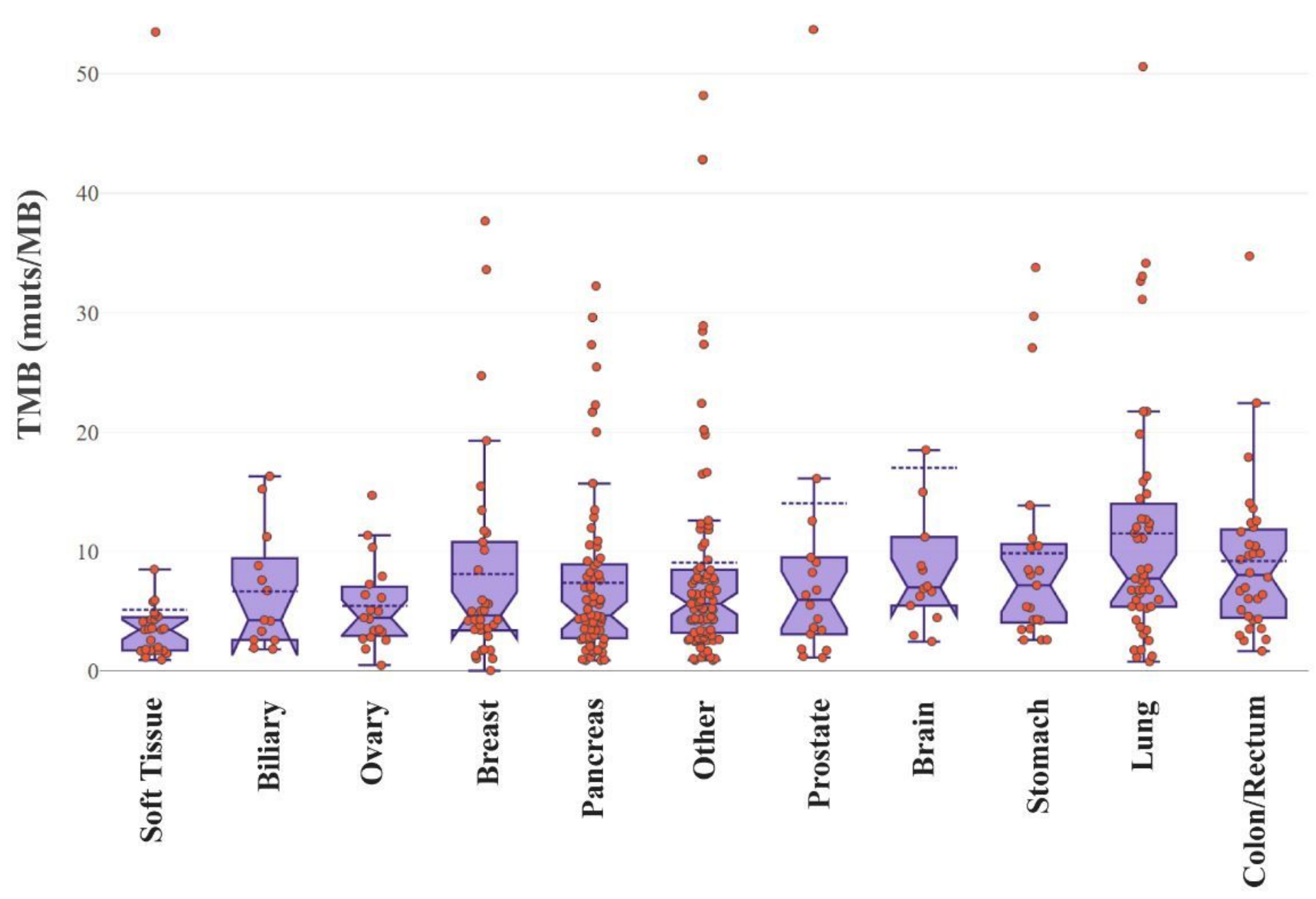

Figure 8

Box plots showing the median TMB values in various tumor types. Three samples with TMB values $>60$ were omitted from the plot for visualization purposes. 


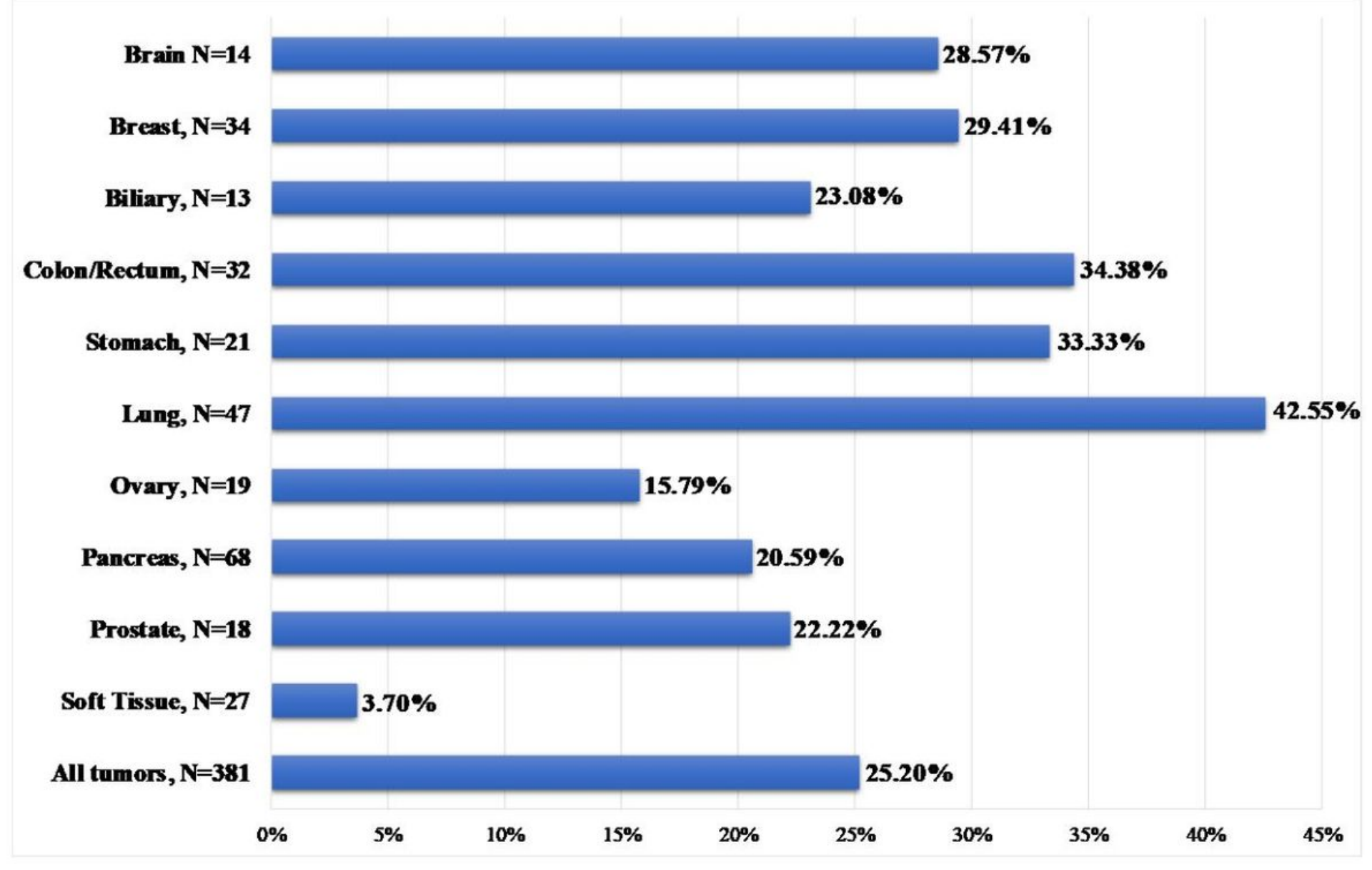

\section{Figure 9}

TMB positivity rate in various histological tumor types. 


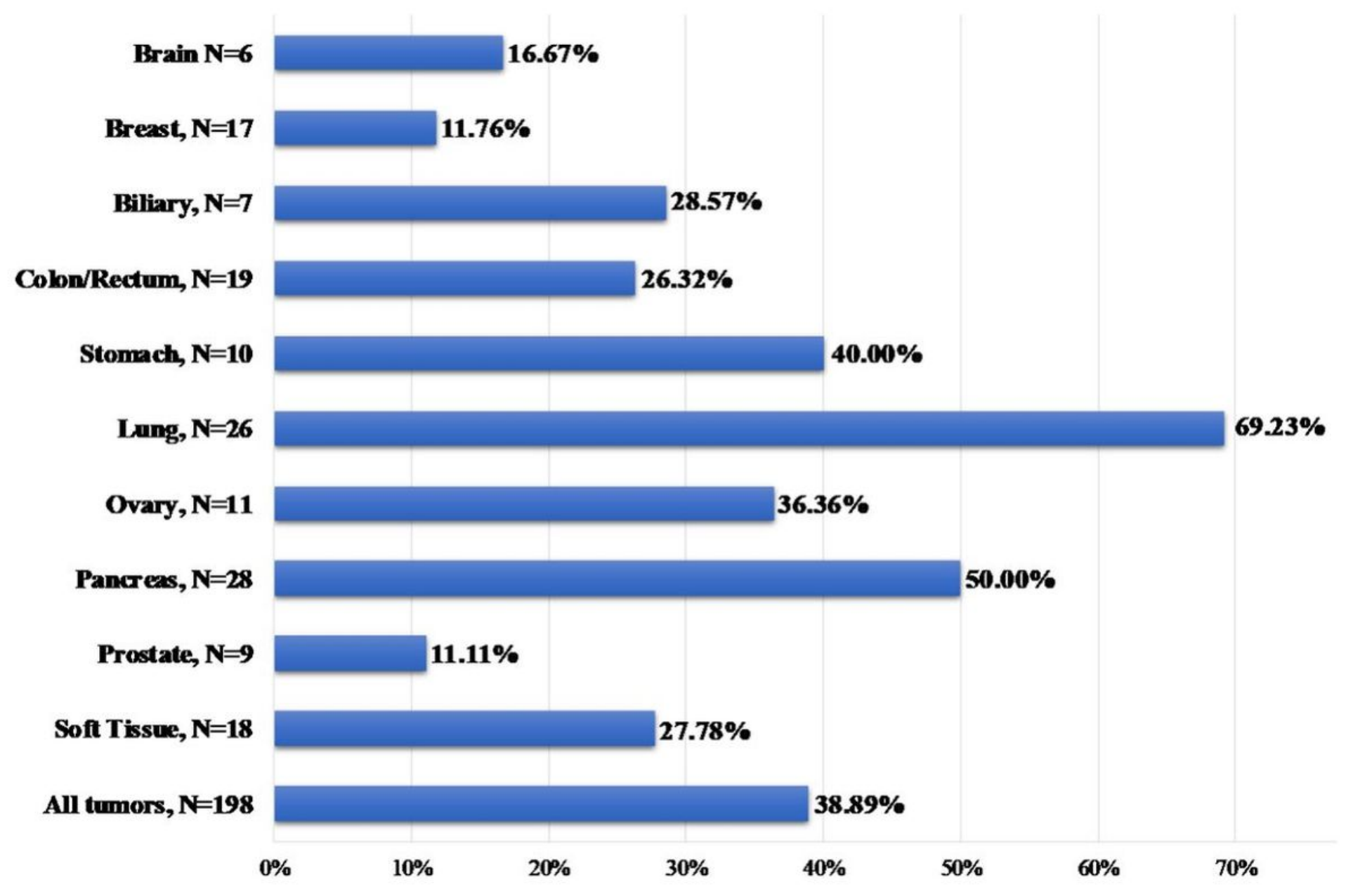

Figure 10

PD-L1 positivity rate in various tumor histological types. 
80

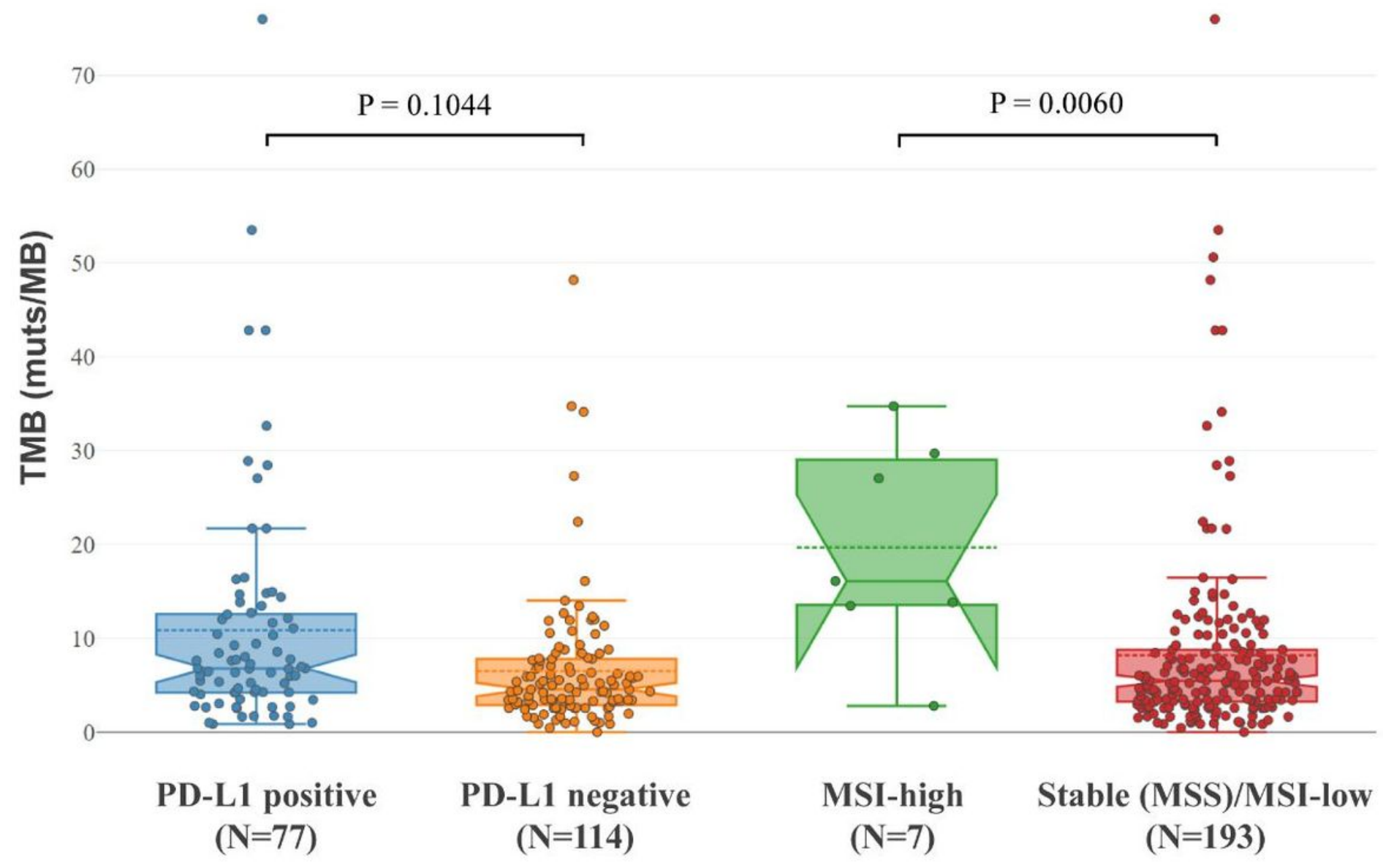

Figure 11

TMB-MSI and TMB-PD-L1 correlation. 


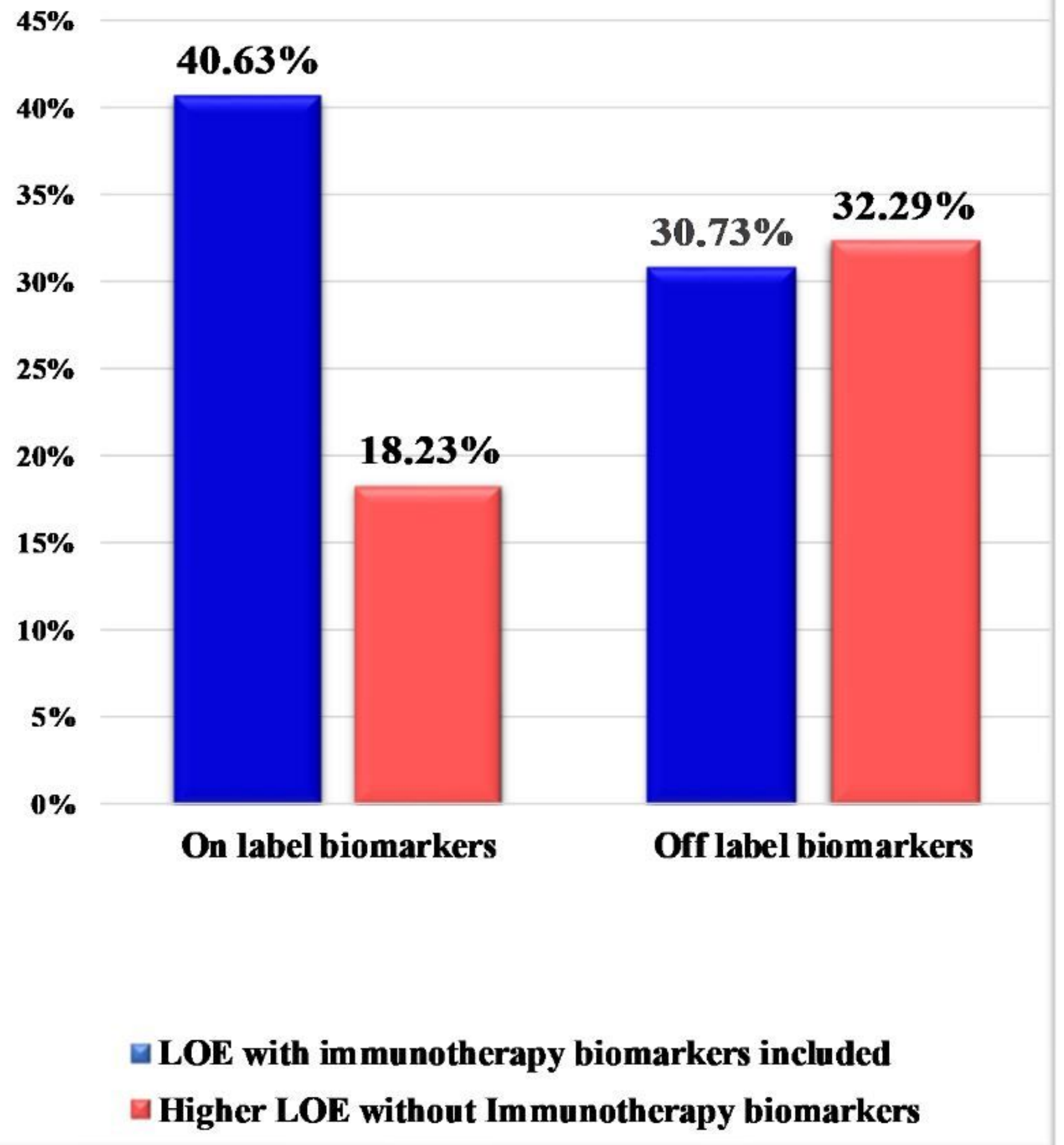

Figure 12

Patients' categorization based on the level of evidence of the most clinically significant variant associated with response, with and without the use of immunotherapy biomarkers. Biomarkers associated with resistance were excluded from this analysis. 


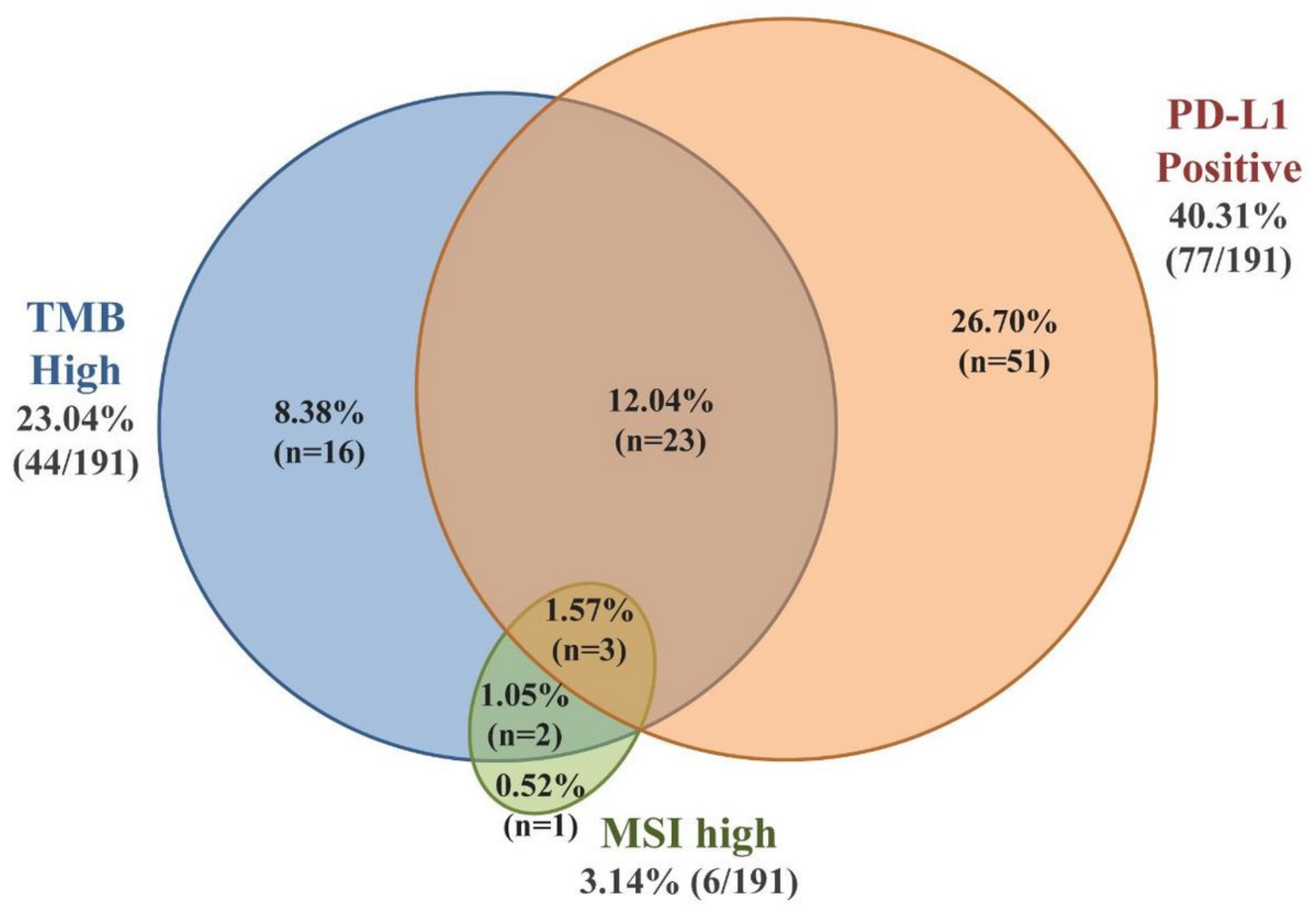

Figure 13

Venn Diagram showing the correlation among the three immunotherapy biomarkers tested (PD-L1, TMB, $\mathrm{MSI})$.

\section{Supplementary Files}

This is a list of supplementary files associated with this preprint. Click to download.

- Additionalfile10new2.xlsx

- Additionalfile9.xlsx

- Additionalfile8.xlsx

- Additionalfile7.tif

- Additionalfile6.tif

- Additionalfile5.tif

- Additionalfile4.tif 
- Additionalfile3.xIsx

- Additionalfile2.xIsx

- Additionalfile1.xlsx 